\title{
Convergence analysis of a vertex-centered finite volume scheme for a copper heap leaching model
}

\author{
Emilio Cariaga, ${ }^{a}$ Fernando Concha, ${ }^{b}$ Iuliu Sorin Pop ${ }^{c}$ \\ and Mauricio Sepúlveda ${ }^{\mathrm{d} * \dagger}$
}

\section{Communicated by $\mathbf{W}$. Wendland}

In this paper a two-dimensional solute transport model is considered to simulate the leaching of copper ore tailing using sulfuric acid as the leaching agent. The mathematical model consists in a system of differential equations: two diffusion-convection-reaction equations with Neumann boundary conditions, and one ordinary differential equation. The numerical scheme consists in a combination of finite volume and finite element methods. A Godunov scheme is used for the convection term and an P1-FEM for the diffusion term. The convergence analysis is based on standard compactness results in $L^{2}$. Some numerical examples illustrate the effectiveness of the scheme. Copyright $\odot 2009$ John Wiley \& Sons, Ltd.

Keywords: compositional flow model; porous media; finite volume method; heap leaching; degenerate parabolic equation; convection-diffusion-reaction system

\section{Introduction}

Heap leaching is a hydro-metallurgical process used for oxides ores with several industrial mining applications including copper, zinc, gold and caliche minerals. In the case of copper production, a leaching model can be considered as a flow of two fluids phases (liquid and gaseous), coupled to the transport of components (sulfuric acid, copper ions, water, air and oxygen) in a porous medium. Systems of diffusion reaction equations modeling fluid flow and physicochemical reactions for the heap leaching process of copper ores have been studied by several authors (see e.g. [1-5]). Subsequently, the advent (in 1980) of the process of agglomeration and acid curing followed by nonflooding or trickle leaching, known as the Thin-Layer (TL) Leaching process, permitted several Chilean mines to be brought into commercial operation. This process had been patented in 1981 by Esteban Domic and described in details in his textbook [6].

There are two phenomena of interest: the fluid flow and the physicochemical reactions. These two phenomena can be studied separately if the leaching process does not influence the flow. In other words, the flow in a heap can be decoupled from the chemistry. In this work we focus on the study of the transport equation and assume that the fluid velocity, the pressure and the saturation are given. Assuming that the pressure in the gaseous phase is constant, the fluid flow is modeled by the Richards equation (see [7]). The heap leaching of copper ore coupled with a two-phase flow model was investigated by Cariaga et al. [8, 9].

\footnotetext{
${ }^{a}$ Department of Mathematical and Physical Sciences, UCTemuco, Ciencias Fisicas, Temuco, Region de la Araucania, Chile

${ }^{b}$ Department of Metallurgical Engineering, University of Concepción, Concepción, Region del Bio-Bio, Chile

${ }^{\prime}$ CASA, Department of Mathematics and Computer Science, Eindhoven University of Technology, Eindhoven, Netherlands

${ }^{d} \mathrm{Cl}^{2} \mathrm{MA}$, Departamento de Ingeniería Matemática, Universidad de Concepción, Concepción, Region del Biobio, Chile

*Correspondence to: Mauricio Sepúlveda, $\mathrm{Cl}^{2} \mathrm{MA}$, Departamento de Ingeniería Matemática, Universidad de Concepción, Concepción, Region del Biobio, Chile.

${ }^{\dagger}$ E-mail: mauricio@ing-mat.udec.cl
} 
Coupled models for reactive porous media flows in unsaturated media are investigated in [10, 11], providing error estimates and the convergence of a Newton scheme for an Euler implicit, mixed finite element discretization.

Numerical methods for the transport equations including the physicochemical reactions phenomena are studied by several authors in different contexts. Specifically, a cell-centered finite volume method is investigated in [12], where numerical simulations of a two-dimensional PDE system for the two-phase flow in a porous medium are given (see also [3]). The work of Muñoz et al. [1] is devoted to the parameter identification and experimental validation of the two-dimensional solute transport model. With respect to a bioleaching model, we mention the work of Casas et al. [2]. Furthermore, Mellado et al. [13, 14] proposed an analytical-numerical method for solving a model of the heap leaching problem.

In the present work, we study the convergence of a vertex-centered finite volume scheme for a system formed by a convectiondiffusion-reaction equation and an ordinary differential equation. This system is complemented with nonhomogeneous flux boundary conditions, which correspond to the physical behavior of the irrigation and infiltration processes in heap leaching. This system of heap leaching transport equations is very similar to that of [12], which was derived from the compositional flow model considered, for instance, in [15]. Specifically we consider the following system:

$$
\begin{aligned}
\partial_{t} u_{1}+\nabla \cdot\left(\alpha_{1}\left(u_{1}\right) \mathbf{v}-\mathbf{D} \nabla \alpha_{1}\left(u_{1}\right)\right) & =\Phi_{1}\left(u_{1}\right) \\
\partial_{t} u_{2}+\nabla \cdot\left(\alpha_{2}\left(u_{2}\right) \mathbf{v}-\mathbf{D} \nabla \alpha_{2}\left(u_{2}\right)\right) & =\Phi_{2}\left(u_{1}, u_{3}\right) \\
\partial_{t}\left(\Psi\left(u_{2}\right)+u_{3}\right) & =\Phi_{3}\left(u_{1}, u_{3}\right)
\end{aligned}
$$

defined in the bounded spatial domain $\Omega \subset \mathbb{R}^{2}$ having a Lipschitz boundary $\partial \Omega$ and representing the transversal cut of the heap, and for $t \in(0, T]$ for some $T>0$. Here $u_{1}, u_{2}$ and $u_{3}$ are unknown concentrations of the sulfuric acid and copper in both liquid and solid phases. Further $\alpha_{1}, \alpha_{2}, \Phi_{1}, \Phi_{2}, \Phi_{3}$ and $\Psi$ are known nonlinear functions expressing the sorption isotherm model (Freundlich isotherm [16] or Langmuir isotherm [17]). The vector $\mathbf{v}$ is the velocity of the fluid flow that is assumed to be known, while $\mathbf{D}$ is the dispersion-diffusion tensor. The diffusion in (1) and (2) may degenerate. Specifically, the physically relevant cases $\alpha_{1}^{\prime}(0)=0$ or $\alpha_{2}^{\prime}(0)=0$ are allowed here.

In this paper we consider a vertex-centered finite volume method for the numerical approximation of the system (1)-(3). It combines the upwind Godunov approach for the convective term, with the $P_{1}$ finite element scheme for the diffusive term. This method was also applied by Afif and Amaziane [18], for a nonlinear and degenerate convection-diffusion equation without reaction term. It is known that for triangular meshes, the discretization of a Laplacian by the piecewise linear conforming finite element method is equivalent to the vertex-centered finite volume method [18, 19], which is also named the box scheme [20, 21], the finite volume element scheme [22], or the control volume finite element scheme [23], see [20, Lemma 3].

In the present heap leaching model, the diffusion is nonlinear and degenerate whenever the volumetric concentration is equal to zero. Therefore the solution may lack regularity, raising also particular difficulties related to the convergence of the approximation. Here, the convergence is obtained by compactness arguments, based on a priori estimates in $L^{\infty}$ and $L^{2}$. In the absence of convective terms, rigorous error estimates are obtained in [24] for a similar scheme.

The paper is organized as follows. In Section 2 we describe the compositional flow model for the volumetric concentration of sulfuric acid in the liquid phase and for the copper ions in the liquid and solid phase. Section 3 gives the mathematical background of the model, including the definition of a weak solution. In Section 4 we introduce the finite volume scheme, where a Godunov scheme is used for the approximation of the convective term, and a standard P1-FEM for the diffusive term. Using $L^{\infty}$ and $L^{2}$ estimates, in Sections 5 and 6 we prove the main convergence results. These are obtained for the sulfuric acid equation, as well as for the copper system. Finally, in Section 7 we present several computational experiments.

\section{The compositional flow model}

\subsection{The heap}

The heap is a homogeneous porous medium consisting of fragmented ore material. It has a trapezoidal shape and is irrigated on the top with a diluted sulfuric acid solution. The copper contained in the ore is desorbed and dissolved into the acid solution. The solute is then transported through the heap. In this way the copper is leached out of the heap and collected at the bottom for a later separation.

The flow in the irrigation process is unsaturated, involving the presence of two phases (liquid and gas). This flow is the transporting mechanism for the copper, see also Figure 1. This situation is modeled by a two-phase-two-component system in the porous medium (the heap). We refer to $[25,26]$ for more details on mathematical models for multiphase, multicomponent flow in porous media.

\subsection{The fluid flow}

In what follows we assume that the pressure of the gaseous phase $p_{n}$ is constant in time and space. Therefore the flow is modeled by the Richards equation $([25,26]$. In a similar context, the two-phase flow model is considered in $[9,27,28])$. We refer to [29] for a more detailed discussion on the two-phase flow equations in the context of heap leaching operations.

Furthermore we assume that the chemical processes do not affect the flow; therefore, the flow problem can be decoupled from the component transport. Denoting by $\phi$ the porosity of the medium, by solving the Richards equation one can determine the liquid saturation $s_{W}$, or the liquid content $\theta_{W}:=\phi s_{W}$, before considering the component transport problem. Consequently, the velocity of the liquid phase $\mathbf{v}_{w}$ is given by the Darcy law. In what follows we assume $s_{W}, \theta_{W}$ and $\mathbf{v}_{w}$ as known quantities depending on time and space. 


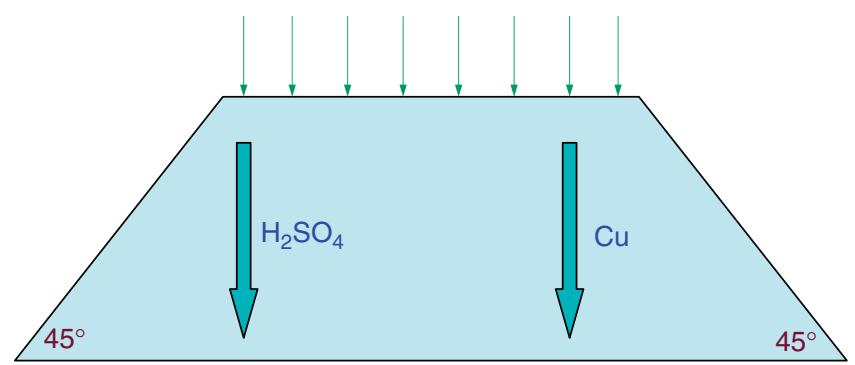

Figure 1. Mathematical domain.

\subsection{Component equations}

The dissolution process is described by the following reaction (see $[1,6,7])$ :

$$
\mathrm{MinOxCu}_{(s)}+2 \mathrm{H}_{(\mathrm{aq})}^{+} \rightarrow \mathrm{Cu}_{(\mathrm{aq})}^{2+}+\mathrm{H}_{2} \mathrm{O}_{(l)}
$$

where $\mathrm{MinOxCu}_{(s)}$ is the copper oxide mineral, which is present in the ore. As a result, two solutes $\left(\mathrm{Cu}^{2+}\right.$ and $\left.\mathrm{H}_{2} \mathrm{SO}_{4}\right)$ are obtained, and these are transported through the unsaturated medium. This process is modeled by the convection-diffusion equation [25]. For the acid solute, we have

$$
\frac{\partial}{\partial t}\left(\phi_{s} \rho_{s} \varphi_{a}\left(\mathrm{C}_{\mathrm{H}_{2} \mathrm{SO}_{4}}\right)+\theta_{w} \mathrm{C}_{\mathrm{H}_{2} \mathrm{SO}_{4}}\right)+\nabla \cdot\left(\mathrm{C}_{\mathrm{H}_{2} \mathrm{SO}_{4}} \mathbf{v}_{w}-\mathbf{D} \nabla \mathrm{C}_{\mathrm{H}_{2} \mathrm{SO}_{4}}\right)+\Phi_{\mathrm{H}_{2} \mathrm{SO}_{4}}=0
$$

where $\mathrm{C}_{\mathrm{H}_{2} \mathrm{SO}_{4}}$ is the volumetric concentration of the sulfuric acid in leach solution, while $\phi_{s}:=1-\phi$ is the volume ratio of the solid part in the heap and $\rho_{\mathrm{s}}$ is the ore bulk density. Further, $\Phi_{\mathrm{H}_{2} \mathrm{SO}_{4}}:=\mu \theta_{W} C_{\mathrm{H}_{2} \mathrm{SO}_{4}}\left[\mathrm{~kg} / \mathrm{m}^{3} \cdot \mathrm{s}\right]$ is the irreversible rate of solute removal from the liquid solution, where $\mu$ is a first-order reaction constant (consumption factor). The function $\varphi_{a}=\varphi_{a}\left(C_{\mathrm{H}_{2} \mathrm{SO}_{4}}\right)$ is the adsorption isotherm of the sulfuric acid. Finally, $\mathbf{D}$ stands for the dispersion-diffusion tensor. This tensor depends on the fluid velocity $\mathbf{v}_{w}$, which we assumed as known. Therefore $\mathbf{D}$ is known here as well. For simplicity we assume that $\mathbf{D}$ is a constant, symmetric and positive-definite tensor.

In the same way, the transport equation for the copper solute is given by

$$
\frac{\partial}{\partial t}\left(\phi_{s} \rho_{s} \varphi_{c}\left(C_{C u}\right)+\theta_{w} C_{C u}\right)+\nabla \cdot\left(C_{C_{u}} \mathbf{v}_{w}-\mathbf{D} \nabla C_{C u}\right)-\Phi_{C u}=0
$$

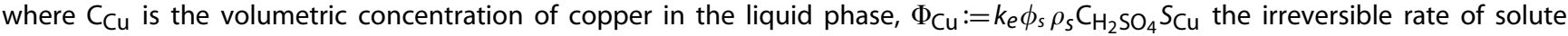
added to the liquid solution, with $k_{e}$ being a first-order kinetic constant, and $S_{\mathrm{Cu}}$ the concentration of copper in the solid phase. The function $\varphi_{\mathrm{c}}=\varphi_{\mathrm{c}}\left(C_{\mathrm{Cu}}\right)$ represents the copper adsorption isotherm.

Here we have neglected the interactions between copper and acid in the adsorption process, that is, we assume that $\varphi_{a}$ depends on $\mathrm{C}_{\mathrm{H}_{2} \mathrm{SO}_{4}}$ only, whereas $\varphi_{\mathrm{c}}$ depends only on $C_{\mathrm{Cu}}$. This does not hold in general for known thermodynamic models of multicomponent two-phase equilibrium isotherms, but can be assumed whenever the concentrations are small.

For the sorption isotherms $\varphi_{\kappa}(0)=0$, with $\kappa=a, c(a=$ acid, $b=$ copper) we assume:

$$
\left(A_{\varphi}\right) \quad \varphi_{\kappa} \text { is a smooth nondecreasing function satisfying } \varphi_{\kappa}(0)=0
$$

Several isotherm functions have been proposed in the literature. The most common ones are the simple linear model $\varphi_{\kappa}(w):=$ $k w$, the Langmuir model $\varphi_{\kappa}(w):=k_{1} w /\left(1+k_{2} w\right)$ or the Freundlich model $\varphi_{\kappa}(w):=k w^{p}, p>0$ (see [15]). More general models of multicomponent isotherms including interaction between molecules can be found in James et al. [30].

Finally, the change in the copper concentration in the solid phase follows the mass balance [1]:

$$
\frac{\partial S_{\mathrm{Cu}}}{\partial t}+k_{e} \theta_{W} C_{\mathrm{H}_{2} \mathrm{SO}_{4}} S_{\mathrm{Cu}}=\frac{\partial\left(\phi_{s} \varphi_{\mathrm{c}}\left(C_{\mathrm{Cu}}\right)\right)}{\partial t}
$$

\subsection{Initial and boundary conditions}

We follow [1] and assume that the boundary of $\Omega$ contains three disjoint parts, $\partial \Omega=\Gamma^{\mathrm{i}} \cup \Gamma^{\mathrm{o}} \cup \Gamma^{\mathrm{t}}$. $\Gamma^{\mathrm{i}}$ is the input boundary (the irrigation zone), where mass balance is assumed

$$
\left\{\begin{array}{l}
\left(C_{\mathrm{H}_{2} \mathrm{SO}_{4}} \mathbf{v}_{W}-\mathbf{D} \nabla C_{\mathrm{H}_{2} \mathrm{SO}_{4}}\right) \cdot \mathbf{n}=C_{\mathrm{H}_{2} \mathrm{SO}_{4}}^{\mathrm{irr}} \mathbf{v}_{W} \cdot \mathbf{n}, \quad \mathbf{x} \in \Gamma^{\mathrm{i}}, t>0 \\
\left(C_{\mathrm{Cu}} \mathbf{v}_{W}-\mathbf{D} \nabla C_{\mathrm{Cu}}\right) \cdot \mathbf{n}=0,
\end{array}\right.
$$

$\mathrm{C}_{\mathrm{H}_{2} \mathrm{SO}_{4}}^{\mathrm{irr}}$ is the sulfuric acid concentration in the irrigation solution. Next, $\Gamma^{\mathrm{O}}$ is the output boundary (the drainage zone), where again by mass balance we have

$$
\left\{\begin{array}{l}
\left(C_{\mathrm{H}_{2} \mathrm{SO}_{4}} \mathbf{v}_{W}-\mathbf{D} \nabla C_{\mathrm{H}_{2} \mathrm{SO}_{4}}\right) \cdot \mathbf{n}=\left(C_{\mathrm{H}_{2} \mathrm{SO}_{4}}-C_{\mathrm{H}_{2} \mathrm{SO}_{4}}^{0}\right) \mathbf{v}_{W} \cdot \mathbf{n}, \\
\left(C_{\mathrm{Cu}} \mathbf{v}_{W}-\mathbf{D} \nabla C_{\mathrm{Cu}}\right) \cdot \mathbf{n}=\left(C_{\mathrm{Cu}}-C_{\mathrm{Cu}}^{0}\right) \mathbf{v}_{W} \cdot \mathbf{n},
\end{array} \quad \mathbf{x} \in \Gamma^{\mathrm{O}}, t>0\right.
$$


The concentrations $\mathrm{C}_{\mathrm{H}_{2} \mathrm{SO}_{4}}^{0}$ and $C_{\mathrm{Cu}}^{0}$ appearing previously are assumed to be constant and equal to the initial concentrations. $\Gamma^{\mathrm{t}}$ is the atmospheric boundary, where we assume that

$$
\left\{\begin{array}{l}
\left(C_{\mathrm{H}_{2} \mathrm{SO}_{4}} \mathbf{v}_{W}-\mathbf{D} \nabla C_{\mathrm{H}_{2} \mathrm{SO}_{4}}\right) \cdot \mathbf{n}=0, \\
\left(C_{\left.\mathrm{Cu} \mathbf{v}_{W}-\mathbf{D} \nabla C_{\mathrm{Cu}}\right) \cdot \mathbf{n}=0,} \quad \mathbf{x} \in \Gamma^{\mathrm{t}}, t>0\right.
\end{array}\right.
$$

Finally, the initial conditions are

$$
C_{\mathrm{H}_{2} \mathrm{SO}_{4}}(\mathbf{x}, 0)=C_{\mathrm{H}_{2} \mathrm{SO}_{4^{\prime}}}^{0} \quad C_{\mathrm{Cu}}(\mathbf{x}, 0)=C_{\mathrm{Cu}^{\prime}}^{0} \quad S_{\mathrm{Cu}}(\mathbf{x}, 0)=S_{\mathrm{Cu}^{\prime}}^{0} \quad \mathbf{x} \in \Omega
$$

where $S_{C \mathrm{C}}^{0}:=\lambda_{\mathrm{C}}^{S} G_{\mathrm{C}}^{S}$, with $\lambda_{\mathrm{C}}^{S}$ being the leachable fraction of the total copper contained in the heap and $G_{\mathrm{C}}^{s}$ is the grade of the ore.

\section{Mathematical framework and variational formulation}

To simplify writing we define new variables. First we denote $S_{C u}$ by $u_{3}$, and $\mathbf{v}_{w}$ by $\mathbf{v}$. Next we use two auxiliary functions $\psi_{1}$ and $\psi_{2}$ to define

$$
\begin{aligned}
& u_{1}(x, t):=\psi_{1}\left(C_{\mathrm{H}_{2} \mathrm{SO}_{4}}(x, t)\right):=\theta_{s} \varphi_{a}\left(C_{\mathrm{H}_{2} \mathrm{SO}_{4}}(x, t)\right)+\theta_{w}(x, t) C_{\mathrm{H}_{2} \mathrm{SO}_{4}}(x, t) \\
& u_{2}(x, t):=\psi_{2}\left(C_{\mathrm{Cu}}(x, t)\right):=\theta_{s} \varphi_{c}\left(C_{\mathrm{Cu}}(x, t)\right)+\theta_{w}(x, t) C_{\mathrm{Cu}}(x, t)
\end{aligned}
$$

where $\theta_{s}=\phi_{s} \rho_{s}$. By the assumption $\left(A_{\varphi}\right)$, the functions $\alpha_{1}\left(u_{1}\right):=\psi_{1}^{-1}\left(u_{i}\right)=C_{\mathrm{H}_{2} \mathrm{SO}_{4}}$ and $\alpha_{2}\left(u_{2}\right):=\psi_{2}^{-1}\left(u_{2}\right)=C_{\mathrm{Cu}}$ are well defined. In this way, for the convergence analysis, we define the Model Problem $A$ in the unknown $u_{1}$ :

$$
\partial_{t} u_{1}+\nabla \cdot\left(\alpha_{1}\left(u_{1}\right) \mathbf{v}-\mathbf{D} \nabla \alpha_{1}\left(u_{1}\right)\right)=-\theta_{w} \mu \alpha_{1}\left(u_{1}\right)
$$

This corresponds to Equation (1) with $\Phi_{1}\left(u_{1}\right)=-\theta_{w} \mu \alpha_{1}\left(u_{1}\right)$. Further, the Model Problem $B$ in the unknowns $u_{2}$ and $u_{3}$ is defined as

$$
\begin{aligned}
\partial_{t} u_{2}+\nabla \cdot\left(\alpha_{2}\left(u_{2}\right) \mathbf{v}-\mathbf{D} \nabla \alpha_{2}\left(u_{2}\right)\right) & =\theta_{w} \rho_{s} k_{e} \alpha_{1}\left(u_{1}\right) u_{3} \\
\frac{\partial u_{3}}{\partial t}+\theta_{w} k_{e} \alpha_{1}\left(u_{1}\right) u_{3} & =\frac{\partial\left(\phi_{s} \varphi_{c}\left(\alpha_{2}\left(u_{2}\right)\right)\right)}{\partial t}
\end{aligned}
$$

This corresponds to the system (2)-(3) with $\Phi_{2}\left(u_{1}, u_{3}\right)=\theta_{w} \rho_{s} k_{e} \alpha_{1}\left(u_{1}\right) u_{3}, \Phi_{3}\left(u_{1}, u_{3}\right)=-\theta_{w} k_{e} \alpha_{1}\left(u_{1}\right) u_{3}$ and $\Psi\left(u_{2}\right)=-\phi_{s} \varphi_{c}\left(\alpha_{2}\left(u_{2}\right)\right)$. Furthermore, the boundary conditions become

$$
\begin{aligned}
& \left(\alpha_{1}\left(u_{1}\right) \mathbf{v}-\mathbf{D} \nabla \alpha_{1}\left(u_{1}\right)\right) \cdot \mathbf{n}=g_{1}\left(x, t, u_{1}\right) \\
& \left(\alpha_{2}\left(u_{2}\right) \mathbf{v}-\mathbf{D} \nabla \alpha_{2}\left(u_{2}\right)\right) \cdot \mathbf{n}=g_{2}\left(x, t, u_{2}\right)
\end{aligned}
$$

for all $x \in \partial \Omega, t>0$. The particular form of the functions $g_{1}$ and $g_{2}$ depends on the boundary type: irrigation, drainage or atmospheric zone. For simplifying the presentation, the analysis below is carried out for the homogeneous case, $g_{1} \equiv 0$ and $g_{2} \equiv 0$.

Finally, the initial conditions are

$$
u_{1}(x, 0)=u_{1}^{0}(x), \quad u_{2}(x, 0)=u_{2}^{0}(x), \quad u_{3}(x, 0)=u_{3}^{0}(x)
$$

for all $x \in \Omega$, with $u_{1}^{0}=\psi_{1}\left(C_{\mathrm{H}_{2} \mathrm{SO}_{4}}^{0}\right), u_{2}^{0}=\psi_{2}\left(C_{\mathrm{Cu}}^{0}\right)$ and $u_{3}^{0}=S_{\mathrm{Cu}}^{0}$.

In the simplified notation, the compositional flow model reduces to finding the solutions $\left(u_{1}, u_{2}, u_{3}\right)$ of the system (11)-(13), with the boundary conditions (14) and (15), and the initial condition (16).

Notice that the Model Problem A is uncoupled from the Model Problem B, and can be solved independently. Consequently, the convergence analysis will be carried out separately. We further remark that the transport equations (11) and (12) could be degenerate, as encountered, for example, in the case of a Freundlich isotherm model $\varphi(u):=c u^{p}$, with $0<p<1($ see $[15,18,31])$. This mathematical property is in agreement with the physical behavior of $u_{1}$ and $u_{2}$ in heap leaching operations.

\subsection{The weak formulation}

Below we use the standard functional spaces $L^{2}(\Omega), H^{1}(\Omega)$ and $H^{-1}(\Omega)$, the dual space of $H_{0}^{1}(\Omega)$. Let $Q_{T}=\Omega \times(0, T]$ and

$$
V:=\left\{v \in C^{1}\left(0, T ; C^{2}(\bar{\Omega})\right) ; v(\cdot ; T)=0\right\}
$$

3.1.1. Model problem A. A weak solution $u_{1}$ of the model problem $\mathrm{A}$ is a function $u_{1}$ with $\partial_{t} u_{1} \in L^{2}\left(0, T ; H^{-1}(\Omega)\right)$ and $\alpha_{1}\left(u_{1}\right) \in$ $L^{2}\left(0, T ; H^{1}(\Omega)\right)$, such that, for all $\phi \in V$

$$
\int_{Q_{T}}\left(u_{1} \partial_{t} \phi+\left(\alpha_{1}\left(u_{1}\right) \mathbf{v}-\mathbf{D}(\mathbf{v}) \nabla \alpha_{1}\left(u_{1}\right)\right) \cdot \nabla \phi-\mu \theta_{W} \alpha_{1}\left(u_{1}\right) \phi\right)+\int_{\Omega} u_{1}^{\circ} \phi^{\circ}=0
$$




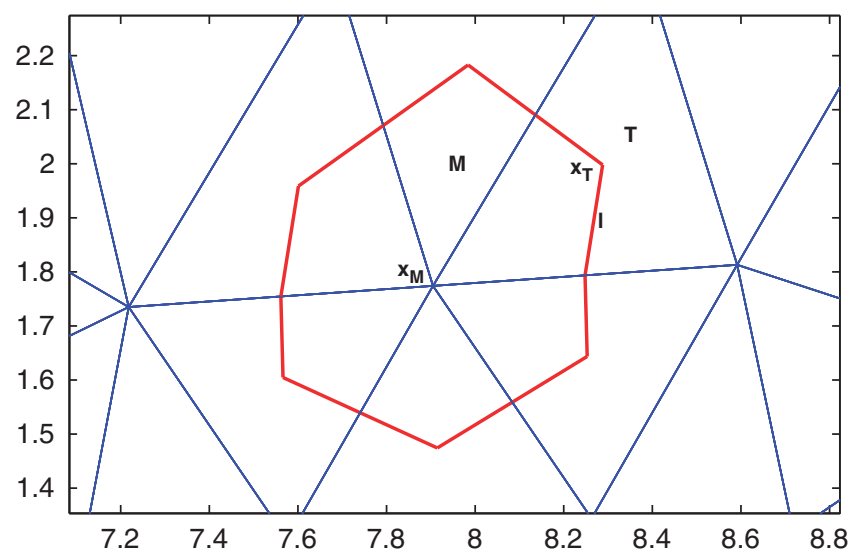

Figure 2. Donald dual mesh.

3.1.2. Model problem B. A weak solution of the model problem B is a pair $\left(u_{2}, u_{3}\right)$, with $\partial_{t} u_{2} \in L^{2}\left(0, T ; H^{-1}(\Omega)\right)$ and $\alpha_{2}\left(u_{2}\right) \in$ $L^{2}\left(0, T ; H^{1}(\Omega)\right)$, such that, for all $\phi \in V$

$$
\int_{Q_{T}}\left(u_{2} \partial_{t} \phi+\left(\alpha_{2}\left(u_{2}\right) \mathbf{v}-\mathbf{D}(\mathbf{v}) \nabla \alpha_{2}\left(u_{2}\right)\right) \cdot \nabla \phi+k_{e} \rho_{s} \theta_{W} \alpha_{1}\left(u_{1}\right) u_{3} \phi\right)+\int_{\Omega} u_{2}^{o} \phi^{\mathrm{o}}=0
$$

as well as $\partial_{t} u_{3} \in L^{2}\left(0, T ; H^{-1}(\Omega)\right)$, such that, for all $\psi \in V$

$$
\int_{Q_{T}} \frac{\partial u_{3}}{\partial t} \psi+\int_{Q_{T}} \theta_{W} k_{e} \alpha_{1}\left(u_{1}\right) u_{3} \psi=\int_{Q_{T}} \frac{\partial\left(\phi_{s} \varphi_{2}\left(\alpha_{2}\left(u_{2}\right)\right)\right)}{\partial t} \psi
$$

\subsection{Existence and uniqueness}

A mathematical analysis of the Model Problem A can be found, for example in [32,33]. For the existence and uniqueness of the Model Problem A, in the context of entropy solutions, we refer to the classical paper of Carrillo [34].

Systems similar to the Model Problem B are encountered as upscaled reactive porous media flows. For the analysis of such systems we refer to [15, 35]. Uniqueness results are obtained in [36]. A model similar to Model Problem B was studied in [37-40].

\section{Finite volume discretization}

The discretization scheme is defined on an admissible mesh, as introduced by Eymard et al. in [41]. Here we restrict our approach to the two-dimensional case.

Definition 1 (Admissible mesh)

Let $\Omega$ be an open-bounded polygonal subset of $\mathbb{R}^{2}$. An admissible finite volume mesh of $\Omega$ is a triplet $(\mathscr{T}, \mathscr{E}, \mathscr{P})$, where $\mathscr{T}$ is a family of open polygonal convex subsets of $\Omega$ called control volumes, $\mathscr{E}$ is a family of subsets of $\bar{\Omega}$ contained in straights of $\mathbb{R}^{2}$ with strictly positive one-dimensional measure, called the edges of the control volumes, and $\mathscr{P}$ is a family of points of $\Omega$ satisfying the following properties:

1. The closure of the union of all the control volumes is $\bar{\Omega}$.

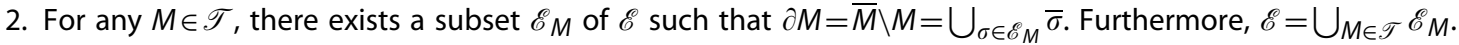

3. For any $(M, L) \in \mathscr{T}^{2}$ with $M \neq L$, either the $(d-1)$-dimensional Lebesgue measure of $\bar{M} \cap \bar{L}$ is 0 or $\bar{M} \cap \bar{L}=\bar{\sigma}$ for some $\sigma \in \mathscr{E}$, which then is denoted by $M \mid L$ or $I$.

4. The family $\mathscr{P}=\left(x_{M}\right)_{M \in \mathscr{T}}$ is such that $x_{M} \in \bar{M}$ (for all $M \in \mathscr{T}$ ) and, if $\sigma=M \mid L=l$, we assumed that $x_{M} \neq x_{L}$, and that the straight line $\mathscr{D}_{M, L}$ going through $x_{M}$ and $x_{L}$ is orthogonal to $M \mid L=l$.

5. For any $\sigma \in \mathscr{E}$ such that $\sigma \subset \partial \Omega$, let $M$ be the control volume such that $\sigma \in \mathscr{E}_{M}$. If $x_{M} \notin \sigma$, let $\mathscr{D}_{M, \sigma}$ be the straight line going through $x_{M}$ and orthogonal to $\sigma$, then the condition $\mathscr{D}_{M, \sigma} \cap \sigma \neq \emptyset$ is assumed; let $y_{\sigma}=\mathscr{D}_{M, \sigma} \cap \sigma$.

In this work, we consider a Donald Dual mesh $\mathscr{T}$ (see [42]) generated by a finite element triangulation $\Lambda:=\left\{T_{i}, i=1, \ldots, N_{e}\right\}$ (see [43]). The barycenter of $T \in \Lambda$ is such that $x_{T}=\bigcap_{M \cap T \neq \varnothing} \partial M \in T$. The family $\mathscr{P}$ is formed by the points $x_{M}:=\bigcap_{T \cap M \neq \varnothing} \partial T \in M$ for each control volume of $\mathscr{T}$. We denote by $I:=\partial M_{i} \cap \partial M_{j} \cap T$ the line segment between the points $x_{T}$ and the midpoint of $\left(x_{M_{i}} x_{M_{j}}\right)$ and let $\mathscr{E}:=\{I \in \partial M \backslash \Gamma \mid M \in \mathscr{T}\}$ (see Figure 2). The triplet $(\mathscr{T}, \mathscr{E}, \mathscr{P})$ is an admissible mesh in the sense of the previous definition.

In the sequel, the following notations are used. The mesh size is defined by $h:=\operatorname{size}(\mathscr{T})=\sup _{M \in \mathscr{T}} \operatorname{diam}(M)$. For any $M \in \mathscr{T},|M|$ is the area of $M$. The set of neighbors of $M$ is denoted by $\mathscr{N}(M)$, that is $\mathscr{N}(M)=\{L \in \mathscr{T} \mid \exists \sigma \in \mathscr{E} M, \bar{\sigma}=\bar{M} \cap \bar{L}\}$. 
The time discretization may be performed with a variable time step; in order to simplify the notations, we consider a constant time step $\Delta t \in(0, T)$. Let $N_{\Delta t} \in \mathbb{N}^{*}$ such that $N_{\Delta t}:=\max \{n \in \mathbb{N} \mid n k<T\}$, and we denote $t_{n}=n \Delta t$, for $n \in\left\{0, \ldots, N_{\Delta t+1}\right\}$.

The discrete unknowns are denoted by $\left\{w_{M}^{n} \mid M \in \mathscr{T}, n \in\left\{0, \ldots, N_{\Delta t}+1\right\}\right\}$; the value $w_{M}^{n}$ is an expected approximation of $w\left(x_{M}, n \Delta t\right)$, where $w \equiv u_{1}, u_{2}$ or $u_{3}$.

\section{Definition 2}

Let $X(\mathscr{T}, \Delta t)$ be the set of functions $w$ from $\Omega \times\left(0,\left(N_{\Delta t}+1\right) \Delta t\right)$ to $\mathbb{R}$ such that there exists a family of real values $\left\{w_{M}^{n} \mid M \in \mathscr{T}, n \in\right.$ $\left.\left\{0, \ldots, N_{\Delta t}+1\right\}\right\}$, with $w(x, t)=w_{M}^{n}$ for a.e. $x \in M, M \in \mathscr{T}$ and for a.e. $t \in[n \Delta t,(n+1) \Delta t)$, with $n \in\left\{0, \ldots, N_{\Delta t}\right\}$.

\subsection{Discretization of Equation (11)}

Integrating (11) over the set $M \times\left[t_{n}, t_{n+1}\right]$ with $M \in \mathscr{T}$, we obtain

$$
\int_{M}\left(u_{1}\left(\cdot, t_{n+1}\right)-u_{1}\left(\cdot, t_{n}\right)\right)+T_{c}-T_{d}=T_{r}
$$

where $T_{\mathrm{c}}:=\sum_{l \in \partial M} \int_{t_{n}}^{t_{n+1}} \int_{l} \alpha_{1}\left(u_{1}\right) \mathbf{v} \cdot \mathbf{n}_{M, l}, T_{\mathrm{d}}:=\sum_{l \in \partial M} \int_{t_{n}}^{t_{n+1}} \int_{l} \mathbf{D}(\mathbf{v}) \nabla \alpha_{1}\left(u_{1}\right) \cdot \mathbf{n}_{M, l}$ and $T_{\mathrm{r}}:=-\mu \int_{M} \int_{t_{n}}^{t_{n+1}} \theta_{W} \alpha_{1}\left(u_{1}\right)$ are the convective, diffusive and reactive terms, respectively, and $\mathbf{n}_{M, l}$ is the outward normal to $l \in \partial M$. The advection term $T_{C}$ is approximated by an upwind Godunov scheme (see [42]).

$$
T_{\mathrm{c}} \approx \sum_{l}|\Delta t||I| \alpha_{1}\left(u_{1, I}^{n+1}\right) \mathbf{v}_{l}^{n} \cdot \mathbf{n}_{M, I}=-\sum_{l}|\Delta t||I|\left(\alpha_{1}\left(u_{1, M_{l}}^{n+1}\right)-\alpha_{1}\left(u_{1, M}^{n+1}\right)\right)\left(-\mathbf{v}_{l}^{n} \cdot \mathbf{n}_{M, I}\right)^{+}
$$

where

$$
u_{1, l}^{n+1}:= \begin{cases}u_{1, M}^{n+1} & \text { if } \mathbf{v}_{l}^{n} \cdot \mathbf{n}_{M, l} \geqslant 0 \\ u_{1, M_{l}}^{n+1} & \text { if } \mathbf{v}_{l}^{n} \cdot \mathbf{n}_{M, l}<0\end{cases}
$$

On the other hand, the diffusive term $T_{\mathrm{d}}$ is approximated by an P1-FEM scheme. Specifically, the standard P1 finite element basis satisfying $\chi_{M_{i}}\left(M_{j}\right)=\delta_{i j}$ gives

$$
T_{\mathrm{d}} \approx \sum_{l} D_{M, l}\left(\alpha_{1}\left(u_{1, M_{l}}^{n+1}\right)-\alpha_{1}\left(u_{1, M}^{n+1}\right)\right)
$$

where $D_{M, l}:=-|T| \nabla \chi_{M_{l}, T} \cdot \mathbf{D}\left(\mathbf{v}_{T}^{n}\right) \nabla \chi_{M, T}$, and $T$ is such that $M \cap T \neq \emptyset$ and $I \in \partial M \cap T$. Finally, the reaction term $T_{\mathrm{r}}$ is approximated by

$$
\int_{M} \int_{t_{n}}^{t_{n+1}} \theta_{w} \alpha_{1}\left(u_{1}\right) \approx|\Delta t||M| \theta_{w, M}^{n+1} \alpha_{1}\left(u_{1, M}^{n+1}\right)
$$

where $\theta_{w, M}^{n+1}$ is the mean value of $\theta_{w}$ over $M \times\left[t_{n}, t_{n+1}\right]$. Using (17)-(19), the finite volume approximation to (11) is defined as

$$
|M|\left(u_{1, j}^{n+1}-u_{1, j}^{n}\right)+\hat{T}_{1 \mathrm{c}}-\hat{T}_{1 \mathrm{~d}}=\hat{T}_{1 \mathrm{r}}
$$

where

$$
\begin{aligned}
& \hat{T}_{1 \mathrm{c}}:=-|\Delta t| \sum_{I}|I|\left(\alpha_{1}\left(u_{1 /}^{n+1}\right)-\alpha_{1}\left(u_{1, j}^{n+1}\right)\right)\left(-\mathbf{v}_{I}^{n} \cdot \mathbf{n}_{j l}\right)^{+} \\
& \hat{T}_{1 \mathrm{~d}}:=|\Delta t| \sum_{l}\left(\alpha_{1}\left(u_{1 /}^{n+1}\right)-\alpha_{1}\left(u_{1, j}^{n+1}\right)\right) D_{j l} \\
& \hat{T}_{1 \mathrm{r}}:=-\mu|\Delta t||M| \theta_{W, M}^{n+1} \alpha_{1}\left(u_{1, M}^{n+1}\right)
\end{aligned}
$$

Here, we have noted $j \equiv M \equiv M_{j}, I \equiv M_{l}$.

\subsection{Discretization of the system (12)-(13)}

As for (20), the finite volume scheme for (12)-(13) reads

$$
\begin{aligned}
&|M|\left(u_{2, j}^{n+1}-u_{2, j}^{n}\right)+\hat{T}_{2 c}-\hat{T}_{2 \mathrm{~d}}=\hat{T}_{2 \mathrm{r}} \\
& \frac{u_{3, j}^{n+1}-u_{3, j}^{n}}{|\Delta t|}+k_{e} \theta_{w j}^{n} \alpha_{1}\left(u_{1, j}^{n}\right) u_{3, j}^{n}=\phi_{s} \frac{\left(\varphi_{2} \circ \alpha_{2}\right)\left(u_{2, j}^{n+1}\right)-\left(\varphi_{2} \circ \alpha_{2}\right)\left(u_{2, j}^{n}\right)}{|\Delta t|}
\end{aligned}
$$

where

$$
\begin{aligned}
& \hat{T}_{2 \mathrm{c}}:=-|\Delta t| \sum_{l}|I|\left(\alpha_{2}\left(u_{2 l}^{n+1}\right)-\alpha_{2}\left(u_{2, j}^{n+1}\right)\right)\left(-\mathbf{v}_{l}^{n} \cdot \mathbf{n}_{j l}\right)^{+} \\
& \hat{T}_{2 \mathrm{~d}}:=|\Delta t| \sum_{l}\left(\alpha_{2}\left(u_{2 l}^{n+1}\right)-\alpha_{2}\left(u_{2, j}^{n+1}\right)\right) D_{j l} \\
& \hat{T}_{2 \mathrm{r}}:=k_{e} \rho_{s}|\Delta t||M| \theta_{w j}^{n} \alpha_{1}\left(u_{1, j}^{n}\right) u_{3, j}^{n}
\end{aligned}
$$


In this section Equation (11) and its corresponding numerical scheme (20) are considered. The convergence analysis follows the main ideas of $[18,41,44]$.

Let us state the following assumptions:

(A1) $\Omega$ is a bounded open polygonal subset of $\mathbb{R}^{2}$.

(A2) $\theta_{W}(x, t) \in L^{\infty}(\Omega \times(0, T))$ is such that $0<\theta_{W}^{-} \leqslant \theta_{W}(x, t) \leqslant \theta_{w}^{+}<1$.

(A3) $\mathbf{D}$ is a bounded, uniformly positive-definite symmetric tensor on $\Omega \times(0, T)$.

(A4) $u_{i}(x, t=0), \in L^{\infty}(\Omega \times(0, T)), i=1,2,3$.

(A5) $\alpha_{i} \in C^{1}\left[0, \infty\left[\right.\right.$, such that $\alpha_{i}^{\prime}(0)=0$ y $\alpha_{i}^{\prime}(s)>0, \forall s>0, i=1,2$. $\alpha_{i}, \alpha_{i}^{\prime} \in L^{\infty}(\Omega \times(0, T)), i=1,2$.

(A6) $\mathbf{v}_{w} \in\left(L^{\infty}(\Omega \times(0, T))^{2}, \nabla \cdot \mathbf{v}_{w}=0, \mathbf{v}_{w}^{n} \rightarrow \mathbf{v}_{w}\right.$ in $L^{2}(\Omega \times(0, T))$.

(A7) $\varphi_{i}, \alpha_{i}, i=1,2$, are Lipschitz continuous with constant $K_{\varphi_{i}}, L_{\alpha_{i}}, i=1,2$, respectively.

Note that the assumption (A5) includes the case of the degenerate parabolic equation, and in particular the porous medium equation.

\section{1. $L^{\infty}$ stability, existence and uniqueness}

The existence of solutions for the scheme (20) is provided by the following lemma (see [45]):

Lemma 1 (Acute angle condition)

Let $\mathscr{A}$ be a finite-dimensional Hilbert space with scalar product $(\cdot, \cdot)$ and norm $\|\cdot\|$, and let $\mathscr{P}$ be a continuous mapping from $\mathscr{A}$ into itself such that for $\|\xi\|=r>0:(\mathscr{P}(\xi), \xi)>0$. Then there exists $\xi \in \mathscr{A}$ with $\|\xi\| \leqslant r$ such that $\mathscr{P}(\xi)=0$.

The $L^{\infty}$ stability, existence and uniqueness for the finite volume scheme (20) is given in

Proposition 1

Under the assumptions (A1)-(A7), and assuming that

$$
\mu L_{\alpha_{1}}|| \theta_{w} \|_{\infty}|\Delta t|<\frac{1}{2}
$$

the scheme (20) is $L^{\infty}$ stable. Additionally, the scheme (20) has a unique solution.

Proof

First, we note that there exists $\xi=\xi\left(u_{1, j}^{n+1}\right)$ such that

$$
\alpha_{1}\left(u_{1 /}^{n+1}\right)-\alpha_{1}\left(u_{1, j}^{n+1}\right)=\alpha_{1}^{\prime}(\xi)\left(u_{1 /}^{n+1}-u_{1, j}^{n+1}\right)
$$

Therefore, the scheme (20) can be written as

$$
u_{1, j}^{n+1}-u_{1, j}^{n}-\frac{|\Delta t|}{|M|} \sum_{l}|l|\left(-\mathbf{v}_{l}^{n} \cdot \mathbf{n}_{j l}\right)^{+} \alpha_{1}^{\prime}(\xi)\left(u_{1 /}^{n+1}-u_{1, j}^{n+1}\right)-\frac{|\Delta t|}{|M|} \sum_{l} D_{j l} \alpha_{1}^{\prime}(\xi)\left(u_{1 l}^{n+1}-u_{1, j}^{n+1}\right)+\mu|\Delta t| \theta_{w j}^{n+1} \alpha_{1}\left(u_{1, j}^{n+1}\right)=0
$$

that is,

$$
u_{1, j}^{n+1}\left\{1+\frac{|\Delta t|}{|M|} \sum_{l} \alpha_{1}^{\prime}(\xi)\left(|l|\left(-\mathbf{v}_{l}^{n} \cdot \mathbf{n}_{j l}\right)^{+}+D_{j l}\right)\right\}-\frac{|\Delta t|}{|M|} \sum_{l} \alpha_{1}^{\prime}(\xi)\left(|l|\left(-\mathbf{v}_{l}^{n} \cdot \mathbf{n}_{j l}\right)^{+}+D_{j l}\right) u_{1 /}^{n+1}+\mu|\Delta t| \theta_{w j}^{n+1} \alpha_{1}\left(u_{1, j}^{n+1}\right)=u_{1, j}^{n}
$$

This last scheme can be written as a system of nonlinear equations

$$
A\left(\mathbf{U}_{1}^{n+1}\right) \mathbf{U}_{1}^{n+1}+\mathbf{b}\left(\mathbf{U}_{1}^{n+1}\right)=\mathbf{U}_{1}^{n}, \quad n \geqslant 0
$$

where for all $i \neq j \in\left\{0, \ldots, N_{s}\right\}$

$$
\begin{aligned}
A_{i i} & :=1+\frac{|\Delta t|}{|M|} \sum_{l \in \partial M_{i}} \alpha_{1}^{\prime}(\xi)\left(|I|\left(-\mathbf{v}_{l}^{n} \cdot \mathbf{n}_{i l}\right)^{+}+D_{i l}\right) \\
A_{i j} & :=-\frac{|\Delta t|}{|M|} \sum_{l \in \partial M_{i} \cap \partial M_{j}} \alpha_{1}^{\prime}(\xi)\left(|/|\left(-\mathbf{v}_{l}^{n} \cdot \mathbf{n}_{i l}\right)^{+}+D_{i l}\right) \\
b_{i} & :=\mu|\Delta t| \theta_{w j}^{n+1} \alpha_{1}\left(u_{1, j}^{n+1}\right)
\end{aligned}
$$

and $\mathbf{U}_{1}^{n+1}:=\left[u_{11}^{n+1}, u_{12}^{n+1}, \ldots, u_{1, j}^{n+1}, \ldots, u_{1 N_{s}}^{n+1}\right]^{\top}$. From system (23), it is possible to define an auxiliary problem: given the applications $\mathbf{b}(\cdot)$ and $A(\cdot)$, and the vector $\mathbf{c}:=\mathbf{U}^{n}$, find a vector $\mathbf{x}$ such that,

$$
\mathbf{F}(\mathbf{x}):=A(\mathbf{x}) \mathbf{x}+\mathbf{b}(\mathbf{x})-\mathbf{c}=\mathbf{0}
$$


$L^{\infty}$-stability: We note that the matrix $A$ is a monotone matrix, that is, we have $A_{i j}^{-1} \geqslant 0$. Furthermore $\left(A_{i j}-\sum_{i \neq j}\left|A_{i j}\right|\right) \geqslant 1$, then $\left\|A^{-1}\right\|_{\infty} \leqslant 1$, where $\|\cdot\|_{\infty}$ is the $I_{\infty}$ matrix norm; hence, there exists a generic constant $C>0$, independent of $h,|\Delta t|$ such that, from system (24),

$$
\|\mathbf{x}\|_{\infty}=\left\|A^{-1}(\mathbf{x}) \mathbf{c}-A^{-1}(\mathbf{x}) \mathbf{b}(\mathbf{x})\right\| \leqslant\left\|A^{-1}(\mathbf{x})\right\|_{\infty}\left(\|\mathbf{c}\|_{\infty}+\|\mathbf{b}(\mathbf{x})\|_{\infty}\right) \leqslant\left\|\mathbf{U}_{1}^{n}\right\|_{\infty}+\mu|\Delta t|\left\|\theta_{w}\right\|_{\infty}\left\|\alpha_{1}\right\|_{\infty} \leqslant C
$$

because an induction argument on $n \geqslant 0$.

Existence: At this point it is enough to verify the assumptions of Lemma 1. Further, (24) becomes

$$
(\mathbf{F}(\mathbf{x}), \mathbf{x})=(A(\mathbf{x}) \mathbf{x}, \mathbf{x})+(\mathbf{b}(\mathbf{x}), \mathbf{x})-(\mathbf{c}, \mathbf{x})
$$

where $(\cdot, \cdot)$ denotes the standard Euclidean inner product. Thus, we have that

$$
(A(\mathbf{x}) \mathbf{x}, \mathbf{x})=(\mathbf{x}, \mathbf{x})+(M(\mathbf{x}) \mathbf{x}, \mathbf{x}) \geqslant\|\mathbf{x}\|^{2}
$$

because $A(\mathbf{x})=I+M(\mathbf{x})$, where $I$ is the identity matrix and $M(\mathbf{x})$ has a positive main diagonal elements exceeding the (column, as well as row) sum of the absolute values of the off-diagonal elements, which are negative or zero. Therefore, $M$ is both, row and column wise diagonal dominant, implying that the inner product $(M(\mathbf{x}) \mathbf{x}, \mathbf{x})$ is never negative. On the other hand, we have that

$$
|(\mathbf{b}(\mathbf{x}), \mathbf{x})| \leqslant \mu L_{\alpha_{1}}\left\|\theta_{w}\right\|_{\infty}\left|\Delta t\|\mid \mathbf{x}\|^{2}\right.
$$

that is, $(\mathbf{b}(\mathbf{x}), \mathbf{x}) \geqslant-\mu L_{\alpha_{1}}\left\|\theta_{w}\right\|_{\infty}|\Delta t|\|\mathbf{x}\|^{2}$. Finally, it is enough to mention that $(\mathbf{c}, \mathbf{x}) \geqslant-\frac{1}{2}\left(\|\mathbf{c}\|^{2}+\|\mathbf{x}\|^{2}\right)$. Now, collecting the above inequalities, choosing $r>0$, as $r^{2}:=\|\mathbf{x}\|^{2}>2\|\mathbf{c}\|^{2}>0$, and assuming that $\mu L_{\alpha_{1}}\left\|\theta_{w}\right\|_{\infty}|\Delta t|<\frac{1}{2}$, we have that $(\mathbf{F}(\mathbf{x}), \mathbf{x})>0$ for $\|\mathbf{x}\|=r>0$. Therefore, by Lemma 1 , there exists $\mathbf{x}$ with $\|\mathbf{x}\| \leqslant r$ such that $\mathbf{F}(\mathbf{x})=\mathbf{0}$.

Uniqueness: Let $\mathbf{U}_{1}^{n+1}=\left(u_{1, j}^{n+1}\right)_{j=0, N_{s}}$ and $\mathbf{V}_{1}^{n+1}=\left(v_{1, j}^{n+1}\right)_{j=0, N_{s}}$ be two solutions of the implicit scheme (1), that is for $j=0, \ldots, N_{s}$,

$$
\begin{aligned}
& u_{1, j}^{n+1}-u_{1, j}^{n}-\frac{|\Delta t|}{|M|} \sum_{l}|l|\left(-\mathbf{v}_{l}^{n} \cdot \mathbf{n}_{j l}\right)^{+}\left(\alpha_{1}\left(u_{1 l}^{n+1}\right)-\alpha_{1}\left(u_{1, j}^{n+1}\right)\right)-\frac{|\Delta t|}{|M|} \sum_{l} D_{j l}\left(\alpha_{1}\left(u_{1 l}^{n+1}\right)-\alpha_{1}\left(u_{1, j}^{n+1}\right)\right)+\mu|\Delta t| \theta_{w j}^{n+1} \alpha_{1}\left(u_{1, j}^{n+1}\right)=0 \\
& v_{1, j}^{n+1}-v_{1, j}^{n}-\frac{|\Delta t|}{|M|} \sum_{l}|l|\left(-\mathbf{v}_{l}^{n} \cdot \mathbf{n}_{j l}\right)^{+}\left(\alpha_{1}\left(v_{1 l}^{n+1}\right)-\alpha_{1}\left(v_{1, j}^{n+1}\right)\right)-\frac{|\Delta t|}{|M|} \sum_{l} D_{j l}\left(\alpha_{1}\left(v_{1 l}^{n+1}\right)-\alpha_{1}\left(v_{1, j}^{n+1}\right)\right)+\mu|\Delta t| \theta_{w j}^{n+1} \alpha_{1}\left(v_{1, j}^{n+1}\right)=0
\end{aligned}
$$

By subtracting, and applying similar arguments to existence case, we obtain for $w_{1 q}^{n+1}:=u_{1 q}^{n+1}-v_{1 q}^{n+1}, q=j, l$,

$$
w_{1, j}^{n+1}\left\{1+\frac{|\Delta t|}{|M|} \sum_{l} \alpha_{1, j}^{\prime}\left(\xi_{j}\right)\left(|/|\left(-\mathbf{v}_{l}^{n} \cdot \mathbf{n}_{j l}\right)^{+}+D_{j l}+\mu|\Delta t| \theta_{w j}^{n+1}\right)\right\}-\frac{|\Delta t|}{|M|} \sum_{l} \alpha_{1 /}^{\prime}\left(\xi_{l}\right)\left(|/|\left(-\mathbf{v}_{l}^{n} \cdot \mathbf{n}_{j l}\right)^{+}+D_{j l}\right) w_{1 /}^{n+1}=0
$$

where $\alpha_{1 q}^{\prime}\left(\xi_{q}\right):=\alpha_{1 q}^{\prime}\left(u_{q}^{n+1}, v_{q}^{n+1}\right), q=j, l$. This last scheme can be written as a system of equations,

$$
\tilde{A}\left(\mathbf{U}_{1}^{n+1}, \mathbf{v}_{1}^{n+1}\right) \mathbf{W}_{1}^{n+1}=\mathbf{0}
$$

where following a similar argument to existence case, we obtain the nonsingularity of the matrix $\tilde{A}$. Therefore, $\mathbf{W}_{1}^{n+1}=$ $\mathbf{U}_{1}^{n+1}-\mathbf{V}_{1}^{n+1}=\mathbf{0}$.

Proposition 2

Consider the scheme (20); assuming (A1)-(A7), there exist the constants $C_{i}>0, i=1,2$, independent of $h$ and $\Delta t$, such that

$$
\begin{array}{r}
\Delta t \sum_{n, l}|l|\left(\alpha_{1}\left(u_{1 l}^{n+1}\right)-\alpha_{1}\left(u_{1, j}^{n+1}\right)\right)^{2}\left|\mathbf{v}_{I}^{n} \cdot \mathbf{n}_{j l}\right| \leqslant C_{1} \\
\Delta t \sum_{n, l}\left(\alpha_{1}\left(u_{1 l}^{n+1}\right)-\alpha_{1}\left(u_{1, j}^{n+1}\right)\right)^{2} D_{j l} \leqslant C_{2}
\end{array}
$$

Proof

Multiplying (20) by $u_{1, j}^{n+1}$ and using $\sum_{j}$, we get

$$
\sum_{j}|M| u_{1, j}^{n+1}\left(u_{1, j}^{n+1}-u_{1, j}^{n}\right)=-\sum_{j} u_{1, j}^{n+1} \hat{T}_{1 \mathrm{c}}+\sum_{j} u_{1, j}^{n+1} \hat{T}_{1 \mathrm{~d}}+\sum_{j} u_{1, j}^{n+1} \hat{T}_{1 \mathrm{r}}
$$

Using $0 \leqslant(a-b)^{2} / 2=a(a-b)-\left(a^{2}-b^{2}\right) / 2$ in the left-hand side of this last equation, we obtain

$$
\frac{1}{2} \sum_{j}|M|\left[\left(u_{1, j}^{n+1}\right)^{2}-\left(u_{1, j}^{n}\right)^{2}\right] \leqslant-\sum_{j} u_{1, j}^{n+1} \hat{T}_{1 \mathrm{c}}+\sum_{j} u_{1, j}^{n+1} \hat{T}_{1 \mathrm{~d}}+\sum_{j} u_{1, j}^{n+1} \hat{T}_{1 \mathrm{r}}
$$

that is,

$$
\sum_{j} u_{1, j}^{n+1} \hat{T}_{1 \mathrm{c}}-\sum_{j} u_{1, j}^{n+1} \hat{T}_{1 \mathrm{~d}} \leqslant-\frac{1}{2} \sum_{j}|M|\left[\left(u_{1, j}^{n+1}\right)^{2}-\left(u_{1, j}^{n}\right)^{2}\right]+\sum_{j} u_{1, j}^{n+1} \hat{T}_{1 \mathrm{r}}
$$


In this last inequality, we estimate the convective term

$$
\sum_{j} u_{1, j}^{n+1} \hat{T}_{1 \mathrm{c}}=\sum_{j} u_{1, j}^{n+1}\left\{-|\Delta t| \sum_{l}|I|\left(\alpha_{1}\left(u_{1 /}^{n+1}\right)-\alpha_{1}\left(u_{1, j}^{n+1}\right)\right)\left(-\mathbf{v}_{l}^{n} \cdot \mathbf{n}_{j l}\right)^{+}\right\} \geqslant \frac{|\Delta t|}{2 \sup \left(\alpha_{1}^{\prime}\right)} \sum_{j, l}|I|\left(-\mathbf{v}_{l}^{n} \cdot \mathbf{n}_{j l}\right)^{+}\left(\alpha_{1}\left(u_{1, j}^{n+1}\right)-\alpha_{1}\left(u_{1 /}^{n+1}\right)\right)^{2}
$$

the diffusive term

$$
-\sum_{j} u_{1, j}^{n+1} \hat{T}_{1 \mathrm{~d}}=\sum_{j} u_{1, j}^{n+1}\left\{|\Delta t| \sum_{l}\left(\alpha_{1}\left(u_{1 /}^{n+1}\right)-\alpha_{1}\left(u_{1, j}^{n+1}\right)\right) D_{j l}\right\} \geqslant \frac{|\Delta t|}{2 \sup \left(\alpha_{1}^{\prime}\right)} \sum_{l \in £_{h}} D_{j, l}\left(\alpha_{1}\left(u_{1, j}^{n+1}\right)-\alpha_{1}\left(u_{1 /}^{n+1}\right)\right)^{2}
$$

and the reactive term

$$
\sum_{j} u_{1, j}^{n+1} \hat{T}_{1 \mathrm{r}}=\sum_{j} u_{1, j}^{n+1}\left\{-\mu|\Delta t||M| \theta_{w, M}^{n+1} \alpha_{1}\left(u_{1, M}^{n+1}\right)\right\} \leqslant \mu|\Delta t|\left\|\theta_{w}\right\|_{\infty}\left\|\alpha_{1}^{\prime}\right\|_{\infty}\left\|\mathbf{U}_{1}^{n+1}\right\|_{\infty}|\Omega|
$$

Then by summing over $n=0, \ldots, N_{\Delta t}$, we deduce that there exists a constant $C>0$, independent of $h$ and $\Delta t$, such that

$$
\begin{aligned}
& \frac{|\Delta t|}{2 \sup \left(\alpha_{1}^{\prime}\right)} \sum_{n, j, l}|l|\left(-\mathbf{v}_{l}^{n} \cdot \mathbf{n}_{j l}\right)^{+}\left(\alpha_{1}\left(u_{1, j}^{n+1}\right)-\alpha_{1}\left(u_{1 l}^{n+1}\right)\right)^{2}+\frac{|\Delta t|}{2 \sup \left(\alpha_{1}^{\prime}\right)} \sum_{n, l \in E_{h}} D_{j, l}\left(\alpha_{1}\left(u_{1, j}^{n+1}\right)-\alpha_{1}\left(u_{1 l}^{n+1}\right)\right)^{2} \\
& \leqslant \frac{1}{2} \sum_{j}|M|\left(\left(u_{1, j}^{0}\right)^{2}-\left(u_{1, j}^{N_{\Delta t}+1}\right)^{2}\right)+\mu T\left\|\theta_{w}\right\|_{\infty}\left\|\alpha_{1}^{\prime}\right\|_{\infty}\left\|\mathbf{U}_{1}^{n+1}\right\|_{\infty}|\Omega| \leqslant C
\end{aligned}
$$

\subsection{Convergence results}

\section{Lemma 2}

Consider the scheme (20). There exists a constant $C>0$, independent of $h$ and $\Delta t$, such that

$$
\sum_{n, j} \Delta t|M|\left(\alpha_{1}\left(u_{1, j}^{n+1}\right)-\alpha_{1}\left(u_{1, j}^{n}\right)\right)^{2}+\sum_{n, l} \Delta t|l|\left(\alpha_{1}\left(u_{1 /}^{n}\right)-\alpha_{1}\left(u_{1, j}^{n}\right)\right)^{2} \leqslant C(h+|\Delta t|)
$$

Proof

Multiplying (20) by $\alpha_{1}\left(u_{1, j}^{n+1}\right)-\alpha_{1}\left(u_{1, j}^{n}\right)$, using $\sum_{j}$ and reordering, we get

$$
\sum_{j}|M|\left(\alpha_{1}\left(u_{1, j}^{n+1}\right)-\alpha_{1}\left(u_{1, j}^{n}\right)\right)\left(u_{1, j}^{n+1}-u_{1, j}^{n}\right)=C_{n+1}-C_{n}+D_{n+1}-D_{n}-R_{n+1}
$$

where for $s \in\{n, n+1\}$,

$$
\begin{aligned}
C_{s} & :=|\Delta t| \sum_{M_{j}} \sum_{l \in \partial M_{j}}|I|\left(\alpha_{1}\left(u_{1 l}^{n+1}\right)-\alpha_{1}\left(u_{1, j}^{n+1}\right)\right) \alpha_{1}\left(u_{1, j}^{s}\right)\left(-\mathbf{v}_{l}^{n} \cdot \mathbf{n}_{j l}\right)^{+} \\
D_{s} & :=|\Delta t| \sum_{M_{j}} \sum_{l \in \partial M_{j}}\left(\alpha_{1}\left(u_{1 l}^{n+1}\right)-\alpha_{1}\left(u_{1, j}^{n+1}\right)\right) \alpha_{1}\left(u_{1, j}^{s}\right) D_{j l} \\
R_{n+1} & :=\mu|\Delta t| \sum_{M_{j}}\left|M_{j}\right| \theta_{w j}^{n+1} \alpha_{1}\left(u_{1, j}^{n+1}\right)
\end{aligned}
$$

Using similar arguments as Afif-Amaziane [18], we estimate first the terms $C_{s}$ and $D_{s}$ : we note that there exist constants $C_{1}>0$ and $C_{2}>0$, independent of $h$ and $\Delta t$, such that $\sum_{n}\left|C_{s}\right| \leqslant C_{1}$ and $\sum_{n}\left|D_{s}\right| \leqslant C_{2}$. For the reactive term $R_{n+1}$, we note that there exists a constant $C_{3}>0$, independent of $h$ and $\Delta t$, such that,

$$
\sum_{n}\left|R_{n+1}\right| \leqslant \mu\left\|\theta_{w}\right\|_{\infty}\left\|\alpha_{1}\right\|_{\infty} \sum_{n}|\Delta t| \sum_{j}\left|M_{j}\right| \leqslant \mu\left\|\theta_{w}\right\|_{\infty}\left\|\alpha_{1}\right\|_{\infty} T|\Omega| \leqslant C_{3}
$$

On the other hand, for the left-hand side in (25), we note that for each real numbers $a$ and $b$ :

$$
\left(\alpha_{1}(b)-\alpha_{1}(a)\right)^{2}=\frac{\alpha_{1}(b)-\alpha_{1}(a)}{b-a}\left(\alpha_{1}(b)-\alpha_{1}(a)\right)(b-a)=\alpha_{1}^{\prime}(\cdot)\left(\alpha_{1}(b)-\alpha_{1}(a)\right)(b-a) \leqslant \sup \left|\alpha_{1}^{\prime}\right|\left(\alpha_{1}(b)-\alpha_{1}(a)\right)(b-a)
$$

Therefore, there exists a constant $C_{4}>0$, such that

$$
\sum_{n, j}\left|M_{j}\right|\left(\alpha_{1}\left(u_{1, j}^{n+1}\right)-\alpha_{1}\left(u_{1, j}^{n}\right)\right)^{2} \leqslant C_{4}
$$

that is,

$$
\sum_{n, j}|\Delta t|\left|M_{j}\right|\left(\alpha_{1}\left(u_{1, j}^{n+1}\right)-\alpha_{1}\left(u_{1, j}^{n}\right)\right)^{2} \leqslant C_{4}|\Delta t|
$$


Finally, by Proposition 2, there exists a constant $C_{5}>0$, such that

$$
|\Delta t| \sum_{n, l}\left(\alpha_{1}\left(u_{1 l}^{n+1}\right)-\alpha_{1}\left(u_{1, j}^{n+1}\right)\right)^{2} D_{-} \leqslant|\Delta t| \sum_{n, l}\left(\alpha_{1}\left(u_{1 l}^{n+1}\right)-\alpha_{1}\left(u_{1, j}^{n+1}\right)\right)^{2} D_{j l} \leqslant C_{5}
$$

that is,

$$
|\Delta t| D_{-} \sum_{n, l}\left(C_{6}|l|\right)\left(\alpha_{1}\left(u_{1 l}^{n+1}\right)-\alpha_{1}\left(u_{1, j}^{n+1}\right)\right)^{2} \leqslant|\Delta t| \sum_{n, l} h\left(\alpha_{1}\left(u_{1 l}^{n+1}\right)-\alpha_{1}\left(u_{1, j}^{n+1}\right)\right)^{2} D_{-} \leqslant h C_{5}
$$

Therefore, there exists a constant $C_{7}>0$ such that

$$
|\Delta t| \sum_{n, j}\left|M_{j}\right|\left(\alpha_{1}\left(u_{1, j}^{n+1}\right)-\alpha_{1}\left(u_{1, j}^{n}\right)\right)^{2}+|\Delta t| \sum_{n, l}|I|\left(\alpha_{1}\left(u_{1 /}^{n+1}\right)-\alpha_{1}\left(u_{1, j}^{n+1}\right)\right)^{2} \leqslant C_{4}|\Delta t|+\frac{h C_{5}}{\left(D_{-}\right) C_{6}} \leqslant(|\Delta t|+h) C_{7}
$$

Lemma 3

Under Assumptions (A1)-(A7), let $\mathscr{T}$ be an admissible mesh in the sense of Definition 1 and $\Delta t \in(0, T)$. Let $u_{1, \mathscr{T}, \Delta t} \in X(\mathscr{T}, \Delta t)$ be given by Definition 2. Let $B_{1}:=\left\|u_{1, \mathscr{T}, \Delta t}\right\|_{L}(\Omega \times(0, T))$ and $L_{\alpha_{1}}$ be the Lipschitz constant of $\alpha_{1}$ on $\left[-B_{1}, B_{1}\right]$. One defines $\tilde{U}_{1}$ by $\tilde{U}_{1}=u_{1, \mathscr{T}, \Delta t}$ a.e. on $\Omega \times(0, T)$, and $\tilde{U}_{1}=0$ a.e. on $\mathbb{R}^{2+1} \backslash \Omega \times(0, T)$. Then, there exists a constant $C>0$, independent of $\tau, h$ and $\Delta t$, such that

$$
\left\|\alpha_{1}\left(\tilde{U}_{1}(\cdot, \cdot+\tau)\right)-\alpha_{1}\left(\tilde{U}_{1}(\cdot, \cdot)\right)\right\|_{L^{2}\left(\mathbb{R}^{2+1}\right)}^{2} \leqslant C|\tau| \quad \forall \tau \in \mathbb{R}
$$

Proof

This proof follows the main ideas of Lemma 18.7 in [41]. Let $\tau \in(0, T)$. As $L_{\alpha_{1}}$ is the Lipschitz constant of $\alpha_{1}$ and $\alpha_{1}$ is nondecreasing, the following inequality holds:

$$
\int_{\Omega \times(0, T-\tau)}\left(\alpha_{1}\left(u_{1, \mathscr{T}, \Delta t}(x, t+\tau)\right)-\alpha_{1}\left(u_{1, \mathscr{T}, \Delta t}(x, t)\right)\right)^{2} \mathrm{~d} x \mathrm{~d} t \leqslant L_{\alpha_{1}} \int_{0}^{T-\tau} A(t) \mathrm{d} t
$$

where, for almost every $t \in(0, T-\tau)$,

$$
A(t)=\int_{\Omega}\left(\alpha_{1}\left(u_{1, \mathscr{T}, \Delta t}(x, t+\tau)\right)-\alpha_{1}\left(u_{1, \mathscr{T}, \Delta t}(x, t)\right)\right)\left(u_{1, \mathscr{T}, \Delta t}(x, t+\tau)-u_{1, \mathscr{T}, \Delta t}(x, t)\right) \mathrm{d} x
$$

Note that the function $A(t)$ may be written as (for more details see proof of Lemma 18.7 in [41])

$$
A(t)=\sum_{M \in \mathscr{T}}\left(\alpha_{1}\left(u_{1 M}^{n_{1}(t)}\right)-\alpha_{1}\left(u_{1 M}^{n_{0}(t)}\right)\right) \sum_{n=1}^{N_{\Delta t}} \chi_{n}(t, t+\tau) m(M)\left(u_{1 M}^{n}-u_{1 M}^{n-1}\right)
$$

where $n_{0}(t), n_{1}(t) \in\left\{0, \ldots, N_{\Delta t}\right\}$ such that $n_{0}(t) \Delta t \leqslant t<\left(n_{0}(t)+1\right) \Delta t$ and $n_{1}(t) \Delta t \leqslant t<\left(n_{1}(t)+1\right) \Delta t$, and $\chi_{n}(t, t+\tau)=1$ if $n \Delta t \in(t, t+\tau]$ and $\chi_{n}(t, t+\tau)=0$ if $n \Delta t \notin(t, t+\tau]$. In (26), the order of summation between $n$ and $M$ is changed and the scheme (20) is used. Hence,

$$
\begin{aligned}
A(t)= & \Delta t \sum_{n=1}^{N_{\Delta t}} \chi_{n}(t, t+\tau)\left[\sum_{M \in \mathscr{T}}\left(\alpha_{1}\left(u_{1 M}^{n_{1}(t)}\right)-\alpha_{1}\left(u_{1 M}^{n_{0}(t)}\right)\right)\left(\sum_{l \in \mathcal{N}(M)}|l|\left(\alpha_{1}\left(u_{1 L}^{n}\right)-\alpha_{1}\left(u_{1 M}^{n}\right)\right)\left(-\mathbf{v}_{l}^{n-1} \cdot \mathbf{n}_{l}\right)^{+}\right)\right. \\
& \left.+\sum_{l \in \mathscr{N}(M)}\left(\alpha_{1}\left(u_{1 L}^{n}\right)-\alpha_{1}\left(u_{1 M}^{n}\right)\right) D_{j l}-\mu|M| \theta_{w j}^{n} \alpha_{1}\left(u_{1, j}^{n}\right)\right]=A_{1}(t)+A_{2}(t)+A_{3}(t)
\end{aligned}
$$

where

$$
\begin{aligned}
& A_{1}(t):=\Delta t \sum_{n=1}^{N_{\Delta t}} \chi_{n}(t, t+\tau) \sum_{M, l}|l|\left(\alpha_{1}\left(u_{1 M}^{n_{1}(t)}\right)-\alpha_{1}\left(u_{1 M}^{n_{0}(t)}\right)\right)\left(\alpha_{1}\left(u_{1 L}^{n}\right)-\alpha_{1}\left(u_{1 M}^{n}\right)\right)\left(-\mathbf{v}_{I}^{n-1} \cdot \mathbf{n}_{l}\right)^{+} \\
& A_{2}(t):=\Delta t \sum_{n=1}^{N_{\Delta t}} \chi_{n}(t, t+\tau) \sum_{M, l}\left(\alpha_{1}\left(u_{1 M}^{n_{1}(t)}\right)-\alpha_{1}\left(u_{1 M}^{n_{0}(t)}\right)\right)\left(\alpha_{1}\left(u_{1 L}^{n}\right)-\alpha_{1}\left(u_{1 M}^{n}\right)\right) D_{j l} \\
& A_{3}(t):=\Delta t \sum_{n=1}^{N_{\Delta t}} \chi_{n}(t, t+\tau) \sum_{M}\left(\alpha_{1}\left(u_{1 M}^{n_{1}(t)}\right)-\alpha_{1}\left(u_{1 M}^{n_{0}(t)}\right)\right)\left(-\mu|M| \theta_{w j}^{n} \alpha_{1}\left(u_{1, j}^{n}\right)\right)
\end{aligned}
$$

For the third term, we have that

$$
\begin{aligned}
\int_{0}^{T-\tau} A_{3}(t) \mathrm{d} t & \leqslant \mu \sum_{n=1}^{N_{\Delta t}} \Delta t\left(\sum_{M \in \mathscr{T}} 2 B_{1} L_{\alpha_{1}}|M|\left\|\theta_{w}\right\|_{\infty}\left\|\alpha_{1}\right\|_{\infty}\right) \int_{0}^{T-\tau} \chi_{n}(t, t+\tau) \mathrm{d} t \\
& \leqslant \tau \mu T|\Omega| 2 B_{1} L_{\alpha_{1}}\left\|\theta_{w}\right\|_{\infty}\left\|\alpha_{1}\right\|_{\infty}
\end{aligned}
$$




$$
\begin{aligned}
\int_{0}^{T-\tau} A_{2}(t) \mathrm{d} t \leqslant & \sum_{n=1}^{N_{\Delta t}} \Delta t \sum_{l}\left(\alpha_{1}\left(u_{1 L}^{n_{0}(t)}\right)-\alpha_{1}\left(u_{1 M}^{n_{0}(t)}\right)\right)\left(\alpha_{1}\left(u_{1 L}^{n}\right)-\alpha_{1}\left(u_{1 M}^{n}\right)\right) D_{j l} \int_{0}^{T-\tau} \chi_{n}(t, t+\tau) \mathrm{d} t \\
& +\sum_{n=1}^{N_{\Delta t}} \Delta t \sum_{l}\left(\alpha_{1}\left(u_{1 L}^{n_{1}(t)}\right)-\alpha_{1}\left(u_{1 M}^{n_{1}(t)}\right)\right)\left(\alpha_{1}\left(u_{1 L}^{n}\right)-\alpha_{1}\left(u_{1 M}^{n}\right)\right) D_{j l} \int_{0}^{T-\tau} \chi_{n}(t, t+\tau) \mathrm{d} t \\
\leqslant & \frac{1}{2} \sum_{n, l} \Delta t\left(\alpha_{1}\left(u_{1 L}^{n_{0}(t)}\right)-\alpha_{1}\left(u_{1 M}^{n_{0}(t)}\right)\right)^{2} D_{j l} \int_{0}^{T-\tau} \chi_{n}(t, t+\tau) \mathrm{d} t+\frac{1}{2} \sum_{n, l} \Delta t\left(\alpha_{1}\left(u_{1 L}^{n_{1}(t)}\right)-\alpha_{1}\left(u_{1 M}^{n_{1}(t)}\right)\right)^{2} D_{j l} \int_{0}^{T-\tau} \chi_{n}(t, t+\tau) \mathrm{d} t \\
& +\sum_{n, l} \Delta t\left(\alpha_{1}\left(u_{1 L}^{n}\right)-\alpha_{1}\left(u_{1 M}^{n}\right)\right)^{2} D_{j l} \int_{0}^{T-\tau} \chi_{n}(t, t+\tau) \mathrm{d} t \\
\leqslant & \frac{1}{2} C \tau+\frac{1}{2} C \tau+C \tau
\end{aligned}
$$

where the constant $C>0$, which is independent of $\tau, k$ and $h$, is from Proposition 2. Finally, applying similar arguments and gathering by edges using the inequality $2 a b \leqslant a^{2}+b^{2} ; a, b \in \mathbb{R}$, this yields

$$
\begin{aligned}
\int_{0}^{T-\tau} A_{1}(t) \mathrm{d} t \leqslant & \frac{1}{2}\left[\sum_{n=1}^{N_{\Delta t}} \Delta t \sum_{l \in \mathscr{N}(M)}|l|\left(\alpha_{1}\left(u_{1 M}^{n_{1}(t)}\right)-\alpha_{1}\left(u_{1 M}^{n_{0}(t)}\right)\right)^{2}\left(-\mathbf{v}_{l}^{n-1} \cdot \mathbf{n}_{j l}\right)^{+}+\sum_{n=1}^{N_{\Delta t}} \Delta t \sum_{l \in \mathcal{N}(M)}|l|\left(\alpha_{1}\left(u_{1 L}^{n_{1}(t)}\right)-\alpha_{1}\left(u_{1 L}^{n_{0}(t)}\right)\right)^{2}\left(\mathbf{v}_{l}^{n-1} \cdot \mathbf{n}_{j l}\right)^{+}\right. \\
& \left.+\sum_{n=1}^{N_{\Delta t}} \Delta t \sum_{l \in \mathscr{N}(M)}|l|\left(\alpha_{1}\left(u_{1 L}^{n}\right)-\alpha_{1}\left(u_{1 M}^{n}\right)\right)^{2}\left|\mathbf{v}_{I}^{n-1} \cdot \mathbf{n}_{j l}\right|\right] \int_{0}^{T-\tau} \chi_{n}(t, t+\tau) \mathrm{d} t \\
\leqslant & \frac{1}{2}\left(C_{1}+C_{2}+C_{3}\right) \tau
\end{aligned}
$$

where $C_{i}>0, i=1,2,3$, are constants, independent of $\tau, \Delta t$ and $h$.

We recall the Kolmogorov compactness theorem in $L^{2}(\Omega)$. The proof of this theorem can be found in [46].

Theorem 1 (Fréchet-Kolmogorov)

Let $\omega$ be an open-bounded set of $\mathbb{R}^{N}, N \geqslant 1,1 \leqslant q \leqslant \infty$ and $A \subset L^{q}(\omega)$. Then, $A$ is relatively compact in $L^{q}(\omega)$ if and only if there exists $\{p(u), u \in A\} \subset L^{q}\left(\mathbb{R}^{N}\right)$ such that

1. $p(u)=u$ a.e. on $\omega$, for all $u \in A$.

2. $\{p(u), u \in A\}$ is bounded in $L^{q}\left(\mathbb{R}^{N}\right)$.

3. $\|p(u)(\cdot+\zeta)-p(u)\|_{L q\left(\mathbb{R}^{N}\right)} \longrightarrow 0$, as $\zeta \longrightarrow 0$, uniformly with respect to $u \in A$.

Now we can prove the main result of this section.

\section{Theorem 2}

Under the assumptions (A1)-(A7), the approximate solution $u_{1 h}$ given by the scheme (20) converges to $u_{1}$ in $L^{2}\left(Q_{T}\right)$ as $h$ and $\Delta t$ go to zero.

Proof

Let us set $\tilde{\alpha}_{1 h}:=\alpha_{1}\left(u_{1 h}\right)$ on $Q_{T}$ and $\tilde{\alpha}_{1 h}:=0$ on $\mathbb{R}^{3} \backslash Q_{T}$. From Propositions 1 and 2 , and Lemma 2 , we have that $\left(\tilde{\alpha}_{1 h}\right) \subseteq L^{\infty}\left(\mathbb{R}^{3}\right) \cap L^{2}\left(\mathbb{R}^{3}\right)$. Then, the first and second items of Theorem 1 are clearly satisfied for $N=d+1, d=2, q=2$ and $\omega=\Omega \times(0, T))$. In order to verify the third hypothesis of Theorem 1, we note that the following inequality is verified:

$$
\left\|\tilde{\alpha}_{1 h}(\cdot+\eta, \cdot+\tau)-\tilde{\alpha}_{1 h}(\cdot, \cdot)\right\|_{L^{2}\left(\mathbb{R}^{2+1}\right)} \leqslant\left\|\tilde{\alpha}_{1 h}(\cdot+\eta, \cdot)-\tilde{\alpha}_{1 h}(\cdot, \cdot)\right\|_{L^{2}\left(\mathbb{R}^{2+1}\right)}+\left\|\tilde{\alpha}_{1 h}(\cdot, \cdot+\tau)-\tilde{\alpha}_{1 h}(\cdot, \cdot)\right\|_{L^{2}\left(\mathbb{R}^{2+1}\right)}
$$

for any $\eta \in \mathbb{R}^{2}$ and $\tau \in \mathbb{R}$. In order to estimate this inequality, we note first that there exists a bound $C(|\eta|)>0$, such that $\| \tilde{\alpha}_{1 h}(\cdot+$ $\eta, \cdot)-\tilde{\alpha}_{1 h}(\cdot, \cdot) \|_{L^{2}\left(\mathbb{R}^{2+1}\right)} \leqslant C(|\eta|)$, and $C(|\eta|) \rightarrow 0$ if $|\eta| \rightarrow 0$ (see [41, Lemma 18.3, p. 851]), and then the first term on the right-hand side is uniformly bounded. On the other hand, applying Lemma 3, there exists a constant $C>0$, independent of $\eta$ and $\tau$, such that $\left\|\tilde{\alpha}_{1 h}(\cdot, \cdot+\tau)-\tilde{\alpha}_{1 h}(\cdot, \cdot)\right\|_{L^{2}\left(\mathbb{R}^{2+1}\right)} \leqslant C \tau$. This yields the compactness of the sequence $\left(\tilde{\alpha}_{1 h}\right)$ in $L^{2}\left(Q_{T}\right)$.

Thus, there exists a subsequence, still denoted by $\left(\tilde{\alpha}_{1 h}\right)$, and there exists $\alpha_{1}^{*} \in L^{2}\left(Q_{T}\right)$ such that $\left(\tilde{\alpha}_{1 h}\right)$ converges to $\alpha_{1}^{*}$ in $L^{2}\left(Q_{T}\right)$. Indeed, as $\left(\tilde{\alpha}_{1 h}\right) \subseteq L^{\infty}\left(Q_{T}\right)$, this convergence holds in $L^{q}\left(Q_{T}\right)$ for all $1 \leqslant q<\infty$. Furthermore, as $\alpha_{1}$ is nondecreasing, we can pass to the limits also in the nonlinearity obtaining $\alpha_{1}^{*}=\alpha_{1}\left(u_{1}^{*}\right)$ and $u_{1}^{*}=\alpha_{1}^{-1}\left(\alpha_{1}^{*}\right)$ (see [41, Theorem 18.2]). Therefore, $\left(\alpha_{1 h}\right)$ converges to $\alpha_{1}\left(u_{1}^{*}\right)$ in $L^{1}\left(Q_{T}\right)$, and even in $L^{p}\left(Q_{T}\right)$ for all $p \in[0, \infty)$. 
It remains to be shown that $u_{1}^{*}$ is a weak solution (see definition in Section 3.1.1). For this, let $v \in V$ be a test function and denote $v_{j}^{n} \equiv v\left(x_{M_{j}}, t_{n}\right)$. Multiplying by $v_{j}^{n}$ in the scheme (20), we obtain by summation

$$
\sum_{n, j}|M||\Delta t| v_{j}^{n} \frac{u_{1, j}^{n+1}-u_{1, j}^{n}}{|\Delta t|}+\sum_{n, j} v_{j}^{n} \hat{T}_{1 \mathrm{c}}=\sum_{n, j} v_{j}^{n} \hat{T}_{1 \mathrm{~d}}+\sum_{n, j} v_{j}^{n} \hat{T}_{1 \mathrm{r}}
$$

that is, $H_{1}+H_{2}=H_{3}+H_{4}$, where

$$
\begin{aligned}
& H_{1}:=-\sum_{j}|M| v_{j}^{o} u_{1, j}^{o}+\sum_{n, j}|\Delta t||M| u_{1, j}^{n+1} \frac{v_{j}^{n}-v_{j}^{n+1}}{|\Delta t|} \\
& H_{2}:=\left.\sum_{n, j}|\Delta t| v_{j}^{n} \sum_{l \in \partial M} \alpha_{1}\left(u_{1}\right)\right|_{l} ^{n+1} \mathbf{v}_{l}^{n} \cdot \mathbf{n}_{j, l}|l| \\
& H_{3}:=\sum_{n, j}|\Delta t| v_{j}^{n} \sum_{l \in \partial M}\left(\alpha 1\left(u_{1 /}^{n+1}\right)-\alpha_{1}\left(u_{1, j}^{n+1}\right)\right) D_{j, l} \\
& H_{4}:=-\sum_{n, j} v_{j}^{n}\left(\mu|\Delta t||M| \theta_{w_{i}}^{n+1} \alpha_{1}\left(u_{1, j}^{n+1}\right)\right)
\end{aligned}
$$

Taking into account the assumptions on the data and using the Lebesgue theorem, it follows that as $h$ and $\Delta t$ tend to 0

$$
H_{1} \longrightarrow-\int_{\Omega} u_{1}^{\circ} v(x, 0)-\int_{Q_{T}} u_{1}^{*} \partial_{t} v
$$

On the other hand, we note that $\mathrm{H}_{2}=\mathrm{H}_{2 a}+\mathrm{H}_{2 b}$, where

$$
H_{2 a}:=\sum_{n, j} \Delta t \sum_{l \in \partial M}\left(\alpha_{1}\left(u_{1}\right)-\alpha_{1}\left(u_{1, j}^{n+1}\right)\right) \mathbf{v}_{l}^{n} \cdot \mathbf{n}_{j l}|l|\left(v_{j}^{n}-v_{l}^{n}\right)
$$

and

$$
H_{2 b}:=-\sum_{n, j}|\Delta t| \alpha_{1}\left(u_{1, j}^{n+1}\right) \sum_{l \in \partial M} \int_{l} v^{n} \mathbf{v}_{l}^{n} \cdot \mathbf{n}_{j l} \mathrm{~d} \sigma
$$

About the estimate of $H_{2 a}$, we note that there exists a constant $C>0$, independent of $h$ and $\Delta t$, such that $\left|H_{2 a}\right| \leqslant C h^{1 / 2}$, that is, $\lim _{h \rightarrow 0}\left|H_{2 a}\right|=0$. On the other hand, we note that

$$
H_{2 b}=-\sum_{n, j}|\Delta t| \int_{M_{j}} \alpha_{1}\left(u_{j}^{n+1}\right) \operatorname{div}\left(\mathbf{v}_{l}^{n} v^{n}\right) \mathrm{d} x=-\sum_{n, j}|\Delta t| \int_{M_{j}} \alpha_{1}\left(u_{j}^{n+1}\right) \mathbf{v}_{l}^{n} \cdot \nabla v^{n} \mathrm{~d} x
$$

Therefore, $H_{2} \longrightarrow-\int_{Q_{T}} \alpha_{1}\left(u_{1}^{*}\right) \mathbf{v} \cdot \nabla v$. About the convergence of $H_{3}$, we note that

$$
\begin{aligned}
H_{3} & =-\sum_{n, j} \Delta t \alpha_{1}\left(u_{1, j}^{n+1}\right) \sum_{l \in \partial M \backslash \Gamma}\left(v_{l}^{n}-v_{j}^{n}\right) D_{j, l} \\
& =-\sum_{n, j} \Delta t \alpha_{1}\left(u_{1, j}^{n+1}\right) \sum_{T \cap M \neq \emptyset} \sum_{l \in \partial M \cap T \backslash \Gamma}\left(D_{T} \nabla v_{T}^{n} \cdot \mathbf{n}_{j, l}|l|\right) \\
& =-\sum_{n, j} \Delta t \alpha_{1}\left(u_{1, j}^{n+1}\right) \sum_{l \in \partial M \backslash \Gamma}\left(D_{l} \nabla v_{l}^{n} \cdot \mathbf{n}_{j, l}|l|\right) \\
& =\sum_{n, j} \Delta t \alpha_{1}\left(u_{1, j}^{n+1}\right) \sum_{l \in \partial M \cap \Gamma}\left(D_{l} \nabla v_{l}^{n} \cdot \mathbf{n}_{j, l}|l|\right)-\sum_{n, j} \Delta t \alpha_{1}\left(u_{1, j}^{n+1}\right) \sum_{l \in \partial M}\left(D_{l} \nabla v_{l}^{n} \cdot \mathbf{n}_{j, l}|l|\right)
\end{aligned}
$$

Therefore,

$$
H_{3} \longrightarrow \int_{J} \int_{\Gamma} \alpha_{1}\left(u_{1}^{*}\right) \mathbf{D}(\mathbf{v}) \nabla v \cdot \mathbf{n} \mathrm{d} s \mathrm{~d} t-\iint_{Q_{T}} \alpha_{1}\left(u_{1}^{*}\right) \operatorname{div}(\mathbf{D}(\mathbf{v}) \nabla v) \mathrm{d} t \mathrm{~d} x
$$

that is, $H_{3} \longrightarrow-\int_{Q_{T}} \mathbf{D}(\mathbf{v}) \nabla \alpha_{1}\left(u_{1}^{*}\right) \cdot \nabla v$. About the convergence of $H_{4}$, it is enough to mention that $H_{4} \longrightarrow-\mu \int_{Q_{T}} \theta_{W} \alpha_{1}\left(u_{1}^{*}\right) v$. Finally, passing to the limit in (24) yields

$$
\left[-\int_{\Omega} u_{1}^{o} v(x, 0)-\int_{Q_{T}} u_{1}^{*} \partial_{t} v\right]-\int_{Q_{T}} \alpha_{1}\left(u_{1}^{*}\right) \mathbf{v} \cdot \nabla v=-\int_{Q_{T}} \mathbf{D}(\mathbf{v}) \nabla \alpha_{1}\left(u_{1}^{*}\right) \cdot \nabla v-\mu \int_{Q_{T}} \theta_{W} \alpha_{1}\left(u_{1}^{*}\right) v
$$

Then, $u_{1}^{*}$ is a weak solution of problem (11) that only admits a unique solution $u_{1}$. Thus the entire sequence $\left(u_{1 h}^{n}\right)$ converges to $u_{1}$, which ends the proof. 


\section{Convergence analysis for model problem B}

In this section we analyze the convergence of the numerical scheme (21)-(22) to a solution of (12)-(13).

6.1. $L^{\infty}$ stability, existence and uniqueness

Proposition 3

Under the assumptions (A1)-(A7) the scheme (22) is $L^{\infty}$ unconditionally stable.

Proof

From (22) and by an induction argument, it is enough to mention that

$$
\left|u_{3, j}^{n+1}\right| \leqslant\left|u_{3, j}^{n}\right|\left(1-k_{e}|\Delta t| \theta_{w j}^{n} \alpha_{1}\left(u_{1, j}^{n}\right)\right)+\phi_{s}\left|\left(\varphi_{2} \circ \alpha_{2}\right)\left(u_{2, j}^{n+1}\right)-\left(\varphi_{2} \circ \alpha_{2}\right)\left(u_{2, j}^{n}\right)\right| \leqslant\left(k_{e} \tau \phi_{s}\left\|\theta_{w}\right\|_{\infty}\left\|\alpha_{1}\right\| \infty\left\|\varphi_{2} \circ \alpha_{2}\right\| \infty\right)\left|u_{3, j}^{n}\right|
$$

where $\left|u_{3, j}^{0}\right| \leqslant C$.

\section{Proposition 4}

Under the assumptions (A1)-(A7), the scheme (21) is $L^{\infty}$ unconditionally stable.

Proof

In this case, the scheme (21) can be written as a system of equations, namely

$$
A\left(\mathbf{U}_{2}^{n+1}\right) \mathbf{U}_{2}^{n+1}=\mathbf{U}_{2}^{n}+\tilde{\mathbf{b}}\left(\mathbf{U}_{1}^{n}, \mathbf{v}_{3}^{n}\right)
$$

with $\tilde{b}_{i}:=k_{e} \rho_{s}|\Delta t| \theta_{w i}^{n} \alpha_{1}\left(u_{1 i}^{n}\right) u_{3 i^{\prime}}^{n}$, the matrix $A$ is the same as in Proposition 1 , and the vectors $\mathbf{U}_{2}^{n}$ and $\tilde{\mathbf{b}}\left(\mathbf{U}_{1}^{n}, \mathbf{v}_{3}^{n}\right)$ are given. In this case the system of nonlinear equations is defined as $\mathbf{F}(\mathbf{x}):=A(\mathbf{x}) \mathbf{x}-\mathbf{c}=0$. The proof follows the same line as that of Proposition 1.

Proposition 5

Under the assumptions (A1)-(A7), there exist constants $C_{i}>0, i=1,2$, independent of $h$ and $\Delta t$, such that

$$
\begin{array}{r}
\Delta t \sum_{n, l}|I|\left(\alpha_{2}\left(u_{2 l}^{n+1}\right)-\alpha_{2}\left(u_{2, j}^{n+1}\right)\right)^{2}\left|\mathbf{v}_{I}^{n} \cdot \mathbf{n}_{j l}\right| \leqslant C_{1} \\
\Delta t \sum_{n, l}\left(\alpha_{2}\left(u_{2 l}^{n+1}\right)-\alpha_{2}\left(u_{2, j}^{n+1}\right)\right)^{2} D_{j l} \leqslant C_{2}
\end{array}
$$

Proof

In a similar way to Proposition 2, we get

$$
\frac{1}{2} \sum_{j}|M|\left[\left(u_{1, j}^{n+1}\right)^{2}-\left(u_{1, j}^{n}\right)^{2}\right] \leqslant-\sum_{j} u_{1, j}^{n+1} \hat{T}_{1 \mathrm{c}}+\sum_{j} u_{1, j}^{n+1} \hat{T}_{1 \mathrm{~d}}+\sum_{j} u_{1, j}^{n+1} \hat{T}_{1 \mathrm{r}}
$$

or equivalently,

$$
\sum_{j} u_{1, j}^{n+1} \hat{T}_{1 \mathrm{c}}-\sum_{j} u_{1, j}^{n+1} \hat{T}_{1 \mathrm{~d}} \leqslant-\frac{1}{2} \sum_{j}|M|\left[\left(u_{1, j}^{n+1}\right)^{2}-\left(u_{1, j}^{n}\right)^{2}\right]+\sum_{j} u_{1, j}^{n+1} \hat{T}_{1 \mathrm{r}}
$$

About the reactive term, there exists a constant $C>0$, independent of $h$ and $\Delta t$ such that,

$$
\sum_{n, j} u_{1, j}^{n+1} \hat{T}_{1 \mathrm{r}}=\sum_{n, j} u_{1, j}^{n+1}\left(k_{e} \rho_{s}|\Delta t|\left|M_{j}\right| \theta_{w j}^{n} \alpha_{1}\left(u_{1, j}^{n}\right) u_{3, j}^{n}\right) \leqslant k_{e} \rho_{s}\left\|\mathbf{U}_{1}^{n+1}\right\|_{\infty}\left\|\theta_{w}\right\|_{\infty}\left\|\alpha_{1}\right\|_{\infty}\left\|\mathbf{v}_{3}^{n}\right\|_{\infty} \sum_{n, j}|\Delta t|\left|M_{j}\right| \leqslant C
$$

In this point the proof follows the same line of Proposition 2.

\subsection{Convergence results}

The following estimate is valid for scheme (21):

Lemma 4

There exists a constant $C>0$, independent of $h$ and $\Delta t$, such that

$$
\sum_{n, j} \Delta t|M|\left(\alpha_{2}\left(u_{2, j}^{n+1}\right)-\alpha_{2}\left(u_{2, j}^{n}\right)\right)^{2}+\sum_{n, l} \Delta t|l|\left(\alpha_{2}\left(u_{2 l}^{n}\right)-\alpha_{2}\left(u_{2, j}^{n}\right)\right)^{2} \leqslant C(h+|\Delta t|)
$$

Proof

The proof follows the ideas for Lemma 2. It is enough mentioning that, for the reaction term, there exists a constant $C_{1}>0$, independent of $h$ and $\Delta t$ such that,

$$
\begin{aligned}
\sum_{n, j}\left|M_{j}\right|\left(\alpha_{2}\left(u_{2, j}^{n+1}\right)-\alpha_{2}\left(u_{2, j}^{n}\right)\right) \hat{T}_{1 \mathrm{r}} & =\sum_{n, j}\left|M_{j}\right|\left(\alpha_{2}\left(u_{2, j}^{n+1}\right)-\alpha_{2}\left(u_{2, j}^{n}\right)\right)\left(k_{e} \rho_{s}|\Delta t| \theta_{w j}^{n} \alpha_{1}\left(u_{1, j}^{n} j u_{3, j}^{n}\right)\right. \\
& \leqslant k_{e} \rho_{s}\left\|\mathbf{U}_{1}^{n+1}\right\|_{\infty}\left\|\theta_{w}\right\|_{\infty}\left\|\alpha_{1}\right\|_{\infty}\left\|\alpha_{2}\right\|_{\infty}\left\|\mathbf{v}_{3}^{n}\right\|_{\infty} \sum_{n, j}|\Delta t|\left|M_{j}\right| \leqslant C_{1}
\end{aligned}
$$


Proposition 6

Under the assumptions (A1)-(A7), there exists a constant $C>0$, independent of $h$ and $\Delta t$, such that

$$
\sum_{n, j}|\Delta t||M|\left(u_{3, j}^{n+1}-u_{3, j}^{n}\right)^{2} \leqslant C(|\Delta t|+h)
$$

Proof

From (22) we get

$$
\sum_{n, j}|\Delta t||M|\left(u_{3, j}^{n+1}-u_{3, j}^{n}\right)^{2} \leqslant T_{1}+T_{2}
$$

Further,

$$
\begin{aligned}
T_{1} & :=\phi_{s} \sum_{n, j}|\Delta t||M|\left|\left(\varphi_{2} \circ \alpha_{2}\right)\left(u_{2, j}^{n+1}\right)-\left(\varphi_{2} \circ \alpha_{2}\right)\left(u_{2, j}^{n}\right)\right|\left|u_{3, j}^{n+1}-u_{3, j}^{n}\right| \\
& \leqslant \phi_{s} \sum_{n, j}|\Delta t||M|\left[\varepsilon\left|\left(\varphi_{2} \circ \alpha_{2}\right)\left(u_{2, j}^{n+1}\right)-\left(\varphi_{2} \circ \alpha_{2}\right)\left(u_{2, j}^{n}\right)\right|^{2}+\frac{1}{4 \varepsilon}\left|u_{3, j}^{n+1}-u_{3, j}^{n}\right|^{2}\right] \\
& \leqslant \phi_{s} \sum_{n, j}|\Delta t||M|\left[\varepsilon K_{\varphi_{2}}\left|\alpha_{2}\left(u_{2, j}^{n+1}\right)-\alpha_{2}\left(u_{2, j}^{n}\right)\right|^{2}+\frac{1}{4 \varepsilon}\left|u_{3, j}^{n+1}-u_{3, j}^{n}\right|^{2}\right]
\end{aligned}
$$

where we can choose $\varepsilon:=\phi_{s} / 2$. Finally we have

$$
T_{2}:=k_{e} \sum_{n, j}|\Delta t|^{2}|M| \theta_{w j}^{n} \alpha_{1}\left(u_{1, j}^{n}\right)\left|u_{3, j}^{n}\right|\left|u_{3, j}^{n+1}-u_{3, j}^{n}\right| \leqslant k_{e}|\Delta t| C_{2}
$$

The conclusion follows straightforwardly.

\section{Proposition 7}

Under the assumptions (A1)-(A7), there exists a constant $C>0$, independent of $h$ and $\Delta t$, such that

$$
\frac{1}{2} \sum_{n, l}|\Delta t||l|\left(u_{3, j}^{n}-u_{3 \mid}^{n}\right)^{2} \leqslant C(|\Delta t|+h)
$$

Proof

From Equation (13)

$$
u_{3, j}(t)=-t k_{e} \theta_{w j}(t) \alpha_{1}\left(u_{1, j}(t)\right) u_{3, j}(t)+\phi_{s}\left(\varphi_{2} \circ \alpha_{2}\right)\left(u_{2, j}\right)(t)+u_{3, j}(0)-\phi_{s}\left(\varphi_{2} \circ \alpha_{2}\right)\left(u_{2, j}\right)(0)
$$

Hence,

$$
\sum_{n, l}|\Delta t||l|\left|u_{3, j}(t)-u_{3 /}(t)\right|^{2}=T_{1}+T_{2}
$$

where

$$
T_{1}:=\phi_{s} \sum_{n, l}|\Delta t||I|\left(\left(\varphi_{2} \circ \alpha_{2}\right)\left(u_{2, j}\right)-\left(\varphi_{2} \circ \alpha_{2}\right)\left(u_{2 l}\right)\right)\left(u_{3, j}-u_{3 l}\right)
$$

and

$$
T_{2}:=-k_{e} \sum_{n, l} t|\Delta t||I|\left(\theta_{w j} \alpha_{1}\left(u_{1, j}\right) u_{3, j}-\theta_{w \mid} \alpha_{1}\left(u_{1 /}\right) u_{3 l}\right)\left(u_{3, j}-u_{3 l}\right)
$$

In order to estimate $T_{1}$, we have

$$
\begin{aligned}
T_{1} & \leqslant \phi_{s} \sum_{n, l}|\Delta t||I|\left[\varepsilon_{1}\left|\left(\varphi_{2} \circ \alpha_{2}\right)\left(u_{2, j}\right)-\left(\varphi_{2} \circ \alpha_{2}\right)\left(u_{2 l}\right)\right|^{2}+\frac{1}{4 \varepsilon_{1}}\left|u_{3, j}-u_{3 \prime}\right|^{2}\right] \\
& \leqslant \phi_{s} \sum_{n, l}|\Delta t||I|\left[\varepsilon_{1} K_{\varphi_{2}}\left|\alpha_{2}\left(u_{2, j}\right)-\alpha_{2}\left(u_{2 l}\right)\right|^{2}+\frac{1}{4 \varepsilon_{1}}\left|u_{3, j}-u_{3 /}\right|^{2}\right]
\end{aligned}
$$

Now, in order to estimate $T_{2}$, applying the identity $a b-c d=b(a-c)+c(b-d)$, for each real numbers $a, b, c, d$, we have

$$
\left(\theta_{w j} \alpha_{1}\left(u_{1, j}\right) u_{3, j}-\theta_{w l} \alpha_{1}\left(u_{1 /}\right) u_{3 l}\right)\left(u_{3, j}-u_{3 l}\right)=u_{3, j} \alpha_{1}\left(u_{1, j}\right)\left(\theta_{w j}-\theta_{w l}\right)+u_{3, j} \theta_{w l}\left(\alpha_{1}\left(u_{1, j}\right)-\alpha_{1}\left(u_{1 l}\right)\right)+\theta_{w l} \alpha_{1}\left(u_{1 /}\right)\left(u_{3, j}-u_{3 l}\right)
$$

Therefore, if we suppose that for each $j, l: \theta_{w j}=\theta_{w l}$, then for $T_{2}$, we get

$$
\begin{aligned}
T_{2} & =-k_{e} \sum_{n, l} t|\Delta t||I|\left\{u_{3, j} \theta_{w}\left(\alpha_{1}\left(u_{1, j}\right)-\alpha_{1}\left(u_{1 /}\right)\right)+\theta_{w} \alpha_{1}\left(u_{1 /}\right)\left(u_{3, j}-u_{3 l}\right)\right\}\left(u_{3, j}-u_{3 l}\right) \\
& =-k_{e} \sum_{n, l} t|\Delta t||I| u_{3, j} \theta_{w}\left(\alpha_{1}\left(u_{1, j}\right)-\alpha_{1}\left(u_{1 / l}\right)\right)\left(u_{3, j}-u_{3 l}\right)-k_{e} \sum_{n, l} t|\Delta t||I| \theta_{w} \alpha_{1}\left(u_{1 / l}\right)\left(u_{3, j}-u_{3 l}\right)^{2}
\end{aligned}
$$


In this last equality, the second term in the right-hand side is exchanged to the left-hand side, obtaining

$$
\begin{aligned}
& \sum_{n, l}|I||\Delta t|\left(u_{3, j}-u_{3 l}\right)^{2} \leqslant \sum_{n, l}|I||\Delta t|\left(1+k_{e} t \theta_{w} \alpha_{1}\left(u_{1 / l}\right)\right)\left(u_{3, j}-u_{3 l}\right)^{2} \\
& \leqslant \phi_{s} \sum_{n, l}|\Delta t||I|\left[\varepsilon_{1} K_{\varphi_{2}}\left|\alpha_{2}\left(u_{2, j}\right)-\alpha_{2}\left(u_{2 l}\right)\right|^{2}+\frac{1}{4 \varepsilon_{1}}\left|u_{3, j}-u_{3 /}\right|^{2}\right]+\sum_{n, l}|\Delta t||I|\left[\varepsilon_{2}\left|u_{3, j}\right|\left|\theta_{w}\right|\left|\alpha_{1}\left(u_{1, j}\right)-\alpha_{1}\left(u_{1 / l}\right)\right|^{2}+\frac{1}{4 \varepsilon_{2}}\left|u_{3, j}-u_{3 \prime}\right|^{2}\right]
\end{aligned}
$$

Therefore it is enough to choose $\varepsilon_{1}=\varepsilon_{2}=1$.

\section{Lemma 5}

Under Assumptions (A1)-(A7), let $\mathscr{T}$ be an admissible mesh in the sense of Definition 1 and $\Delta t \in(0, T)$. Let $u_{3, \mathscr{T}, \Delta t} \in X(\mathscr{T}, \Delta t)$ be given by Definition 2 . One defines $\tilde{u}_{3}$ by $\tilde{u}_{3}=u_{3, \mathscr{T}, \Delta t}$ a.e. on $\Omega \times(0, T)$, and $\tilde{u}_{3}=0$ a.e. on $\mathbb{R}^{2+1} \backslash \Omega \times(0, T)$. Then, there exists a constant $C>0$, independent of $\tau, h$ and $\Delta t$, such that

$$
\left\|\tilde{u}_{3}(\cdot, \cdot+\tau)-\tilde{u}_{3}(\cdot, \cdot)\right\|_{L^{2}\left(\mathbb{R}^{2+1}\right)}^{2} \leqslant C|\tau| \quad \forall \tau \in \mathbb{R}
$$

Proof

We start by noticing that

$$
\int_{\Omega \times(0, T-\tau)}\left(\tilde{u}_{3}(x, t+\tau)-\tilde{u}_{3}(x, t)\right)^{2} \mathrm{~d} t=\int_{0}^{T-\tau} A(t) \mathrm{d} t
$$

where

$$
\begin{aligned}
A(t) & :=\int_{\Omega}\left(\tilde{u}_{3}(x, t+\tau)-\tilde{u}_{3}(x, t)\right)^{2} \mathrm{~d} x \\
& =\sum_{M \in \mathscr{T}}|M|\left(u_{3, j}^{n_{1}(t)}-u_{3, j}^{n_{0}(t)}\right)^{2} \\
& =\sum_{M \in \mathscr{T}}|M|\left(u_{3, j}^{n_{1}(t)}-u_{3, j}^{n_{0}(t)}\right)\left(u_{3, j}^{n_{1}(t)}-u_{3, j}^{n_{0}(t)}\right) \\
& =\sum_{M \in \mathscr{T}}\left(u_{3, j}^{n_{1}(t)}-u_{3, j}^{n_{0}(t)}\right) \sum_{n=n_{0}(t)+1}^{n_{1}(t)}|M|\left(u_{3, j}^{n}-u_{3, j}^{n-1}\right) \\
& =\sum_{M \in \mathscr{T}}\left(u_{3, j}^{n_{1}(t)}-u_{3, j}^{n_{0}(t)}\right) \sum_{n=1}^{N_{\Delta t}} \chi_{n}(t, t+\tau)|M|\left(u_{3, j}^{n}-u_{3, j}^{n-1}\right) \\
& =A_{1}(t)+A_{2}(t)
\end{aligned}
$$

with

$$
\begin{aligned}
& A_{1}(t):=\sum_{M \in \mathscr{T}}\left(u_{3, j}^{n_{1}(t)}-u_{3, j}^{n_{0}(t)}\right) \sum_{n=1}^{N_{\Delta t}} \chi_{n}(t, t+\tau)|M|\left[-\Delta t k_{e} \theta_{w j}^{n} \alpha_{1}\left(u_{1, j}^{n}\right) u_{3, j}^{n}\right] \\
& A_{2}(t):=\sum_{M \in \mathscr{T}}\left(u_{3, j}^{n_{1}(t)}-u_{3, j}^{n_{0}(t)}\right) \sum_{n=1}^{N_{\Delta t}} \chi_{n}(t, t+\tau)|M| \phi_{s}\left[\left(\varphi_{2} \circ \alpha_{2}\right)\left(u_{2, j}^{n+1}\right)-\left(\varphi_{2} \circ \alpha_{2}\right)\left(u_{2, j}^{n}\right)\right]
\end{aligned}
$$

Thus, we estimate the first term

$$
\begin{aligned}
\int_{0}^{T-\tau} A_{1}(t) \mathrm{d} t & \leqslant \sum_{n=1}^{N_{\Delta t}} \Delta t\left[\sum_{M \in(\mathscr{T})}\left(u_{3, j}^{n_{1}(t)}-u_{3, j}^{n_{0}(t)}\right)|M| k_{e} \theta_{w j}^{n} \alpha_{1}\left(u_{1, j}^{n}\right) u_{3, j}^{n} \int_{0}^{T-\tau} \chi_{n}(t, t+\tau) \mathrm{d} t\right] \\
& \leqslant\left(2 T\left\|u_{3}\right\|_{\infty}|\Omega| k_{e}\left\|\theta_{w}\right\|_{\infty}\left\|\alpha_{1}\right\|_{\infty}\left\|u_{3}\right\|_{\infty}\right) \tau \\
& \leqslant C \tau
\end{aligned}
$$

Finally, with the same argument, we deduce that there exists a constant $C>0$ such that $\int_{0}^{T-\tau} A_{2}(t) \mathrm{d} t \leqslant C \tau$.

\section{Theorem 3}

Under the assumptions (A1)-(A7) the approximate solution $\left(u_{2 h}, u_{3 h}\right)$ given by the scheme (21)-(22) converges to $\left(u_{2}, u_{3}\right)$ in $L^{2}\left(Q_{T}\right) \times$ $L^{2}\left(Q_{T}\right)$ as $h$ and $\Delta t$ tend to zero.

\section{Proof}

The proof follows the ideas for Theorem 2. In order to prove the convergence of $u_{2 h}:=u_{2 h}(x, t):=u_{2 M}^{n}$ and $u_{3 h}:=u_{3 h}(x, t):=u_{3 M^{\prime}}^{n}$ in $M \times\left[t_{n}, t_{n+1}\right]$, toward the weak solution defined in Section (3.1.2), we will pass to the limit in the scheme (21)-(22). 
Convergence of $u_{2 h}$ : Let us set $\tilde{\alpha}_{2 h}:=\alpha_{2}\left(u_{2 h}\right)$ on $Q_{T}$ and $\tilde{\alpha}_{2 h}:=0$ on $\mathbb{R}^{3} \backslash Q_{T}$. From Propositions 4 and 5 , and Lemma 4 , we have that $\left(\tilde{\alpha}_{2 h}\right) \subseteq L^{\infty}\left(\mathbb{R}^{3}\right) \cap L^{2}\left(\mathbb{R}^{3}\right)$. Then, applying Theorem 1 and Lemma 4 , we have that $\left(\tilde{\alpha}_{2 h}\right)$ is relatively compact in $L^{2}\left(Q_{T}\right)$. Thus, there exists a subsequence, still denoted by $\left(\tilde{\alpha}_{2 h}\right)$, and there exists $\alpha_{2}^{*} \in L^{2}\left(Q_{T}\right)$ such that $\left(\tilde{\alpha}_{2 h}\right)$ converges to $\alpha_{2}^{*}$ in $L^{2}\left(Q_{T}\right)$. Indeed, as $\left(\tilde{\alpha}_{2 h}\right) \subseteq L^{\infty}\left(Q_{T}\right)$, this convergence holds in $L^{q}\left(Q_{T}\right)$ for all $1 \leqslant q<\infty$. Furthermore, since $\alpha_{2}$ is nondecreasing, Theorem 18.2 in [41] gives that $\alpha_{2}^{*}=\alpha_{2}\left(u_{2}^{*}\right)$, that is $u_{2}^{*}=\alpha_{2}^{-1}\left(\alpha_{2}^{*}\right)$. Therefore, $\left(\alpha_{2 h}\right)$ converges to $\alpha_{2}\left(u_{2}^{*}\right)$ in $L^{1}\left(Q_{T}\right)$, and even in $L^{p}\left(Q_{T}\right)$ for all $p \in[0, \infty)$.

Convergence of $u_{3 h}$ : Let us set $\tilde{u}_{3 h}:=u_{3 h}$ on $Q_{T}$ and $\tilde{u}_{3 h}:=0$ on $\mathbb{R}^{3} \backslash Q_{T}$. From Propositions 3 and 6 , and Lemma 7, we have that $\left(\tilde{u}_{3 h}\right) \subseteq L^{\infty}\left(\mathbb{R}^{3}\right) \cap L^{2}\left(\mathbb{R}^{3}\right)$. Then, the first and second items of Theorem 1 are clearly satisfied with $N=d+1, d=2, q=2$ and $\omega=\Omega \times(0, T)$. On the other hand, for $\eta \in \mathbb{R}^{2}$ and $\tau \in \mathbb{R}$, we have

$$
\left\|\tilde{u}_{3 h}(\cdot+\eta, \cdot+\tau)-\tilde{u}_{3 h}(\cdot, \cdot)\right\|_{L^{2}\left(\mathbb{R}^{2+1}\right)} \leqslant\left\|\tilde{u}_{3 h}(\cdot+\eta, \cdot)-\tilde{u}_{3 h}(\cdot, \cdot)\right\|_{L^{2}\left(\mathbb{R}^{2+1}\right)}+\left\|\tilde{u}_{3 h}(\cdot, \cdot+\tau)-\tilde{u}_{3 h}(\cdot, \cdot)\right\|_{L^{2}\left(\mathbb{R}^{2+1}\right)}
$$

About the first term of the right-hand side of this last inequality, there exists a bound $C(|\eta|)>0$, such that $\| \tilde{u}_{3 h}(\cdot+\eta, \cdot)-$ $\tilde{u}_{3 h}(\cdot, \cdot) \|_{L^{2}\left(\mathbb{R}^{2+1}\right)} \leqslant C(|\eta|)$ and $C(|\eta|) \rightarrow 0$ if $|\eta| \rightarrow 0$ (see [41, Lemma 18.3]). On the other hand, there exists a constant $C>0$, independent of $\eta$ and $\tau$, such that (Lemma 5)

$$
\left\|\tilde{u}_{3 h}(\cdot, \cdot+\tau)-\tilde{u}_{3 h}(\cdot, \cdot)\right\|_{L^{2}\left(\mathbb{R}^{2+1}\right)} \leqslant C \tau
$$

This yields the compactness of the sequence $\left(\tilde{u}_{3 h}\right)$ in $L^{2}\left(Q_{T}\right)$, that is, $\left(\tilde{u}_{3 h}\right)$ is relatively compact in $L^{2}\left(Q_{T}\right)$. Therefore, there exists a subsequence, still denoted by $\left(\tilde{u}_{3 h}\right)$, and there exists $u_{3}^{*} \in L^{2}\left(Q_{T}\right)$ such that $\left(u_{3 h}\right)$ converges to $u_{3}^{*}$ in $L^{2}\left(Q_{T}\right)$. Indeed, as $\left(u_{3 h} \subseteq L^{\infty}\left(Q_{T}\right)\right.$, this convergence holds in $L^{q}\left(Q_{T}\right)$ for all $1 \leqslant q<\infty$. Therefore, $\left(u_{3 h}\right)$ converges to $u_{3}^{*}$ in $L^{1}\left(Q_{T}\right)$, and even in $L^{p}\left(Q_{T}\right)$ for all $p \in[0, \infty)$.

It remains to be shown that $\left(u_{2}^{*}, u_{3}^{*}\right)$ is a weak solution. Because, the proof follows the same lines of Theorem 2 , it is enough to mention that

$$
\sum_{n, j} v_{j}^{n} \hat{T}_{2 r}=\sum_{n, j} v_{j}^{n}\left\{k_{e} \rho_{s}|\Delta t||M| \theta_{w j}^{n} \alpha_{1}\left(u_{1, j}^{n}\right) u_{3, j}^{n}\right\} \rightarrow \int_{Q_{T}} k_{e} \rho_{s} \theta_{w} \alpha_{1}\left(u_{1}\right) u_{3} v
$$

\section{Computational experiments}

In this section we present some two-dimensional numerical results provided by the scheme (20)-(22) for the model discussed in the introduction. We consider a trapezoidal heap $3 \mathrm{~m}$ high and having a length of $15 \mathrm{~m}$ at the bottom (see Figure 1).

A linear function $\varphi_{\mathrm{c}}=k_{\mathrm{d}} C_{\mathrm{Cu}}$ is assumed for the copper adsorption isotherm. Otherwise, we assume that the acid adsorption isotherm is null, that is $\varphi_{a}(c) \equiv 0$.

The parameter values are of the same order of magnitude as those reported in $[1,7]$. First, we choose a velocity vector in the direction of the gravity force (downward direction) with a magnitude given by $\mathbf{u}_{W}=(18 / 36) \cdot 10^{-5} \mathrm{~m} / \mathrm{s}$, and saturation parameter value equal to $s_{W}=0.7(-)$; both parameters correspond to a mean of the numerical results reported in [7, 12]. Here, the porosity is given by $\phi=0.33(-)$ and the consumption factor is $\mu=10^{-5}(1 / \mathrm{s})$; the rest of the parameters are the same reported in [1,7], that is, $k_{e}=8.3 \cdot 10^{-7} \mathrm{~m}^{3} / \mathrm{kg} \cdot \mathrm{s}, k_{\mathrm{d}}=8.67 \cdot 10^{-5} \mathrm{~m}^{3} / \mathrm{kg}, \rho_{\mathrm{s}}=1800 \mathrm{~kg} / \mathrm{m}^{3}, \lambda_{\mathrm{c}}^{\mathrm{s}}=0.62(-), G_{\mathrm{C}}^{s}=2.9 \cdot 10^{-3} \mathrm{~kg} / \mathrm{kg}, C_{\mathrm{H}_{2} \mathrm{SO}}^{0}=10\left(\mathrm{~kg} / \mathrm{m}^{3}\right)$, $C_{\mathrm{Cu}}^{0}=0 \mathrm{~kg} / \mathrm{m}^{3}$ and $\mathrm{C}_{\mathrm{H}_{2} \mathrm{SO}_{4}}^{\mathrm{irr}}=40 \mathrm{~kg} / \mathrm{m}^{3}$.

For the discretization parameter, we consider the following: we choose a fixed step time given by $\Delta t=1$ day, and we consider three different meshes with different refinement: the first one, which is the coarsest mesh, is given by $h=0.9226 \mathrm{~m}$ ( 192 triangles); the second one is given by $h=0.4613 \mathrm{~m}$ (768 triangles); and the finest one is given by $h=0.2306 \mathrm{~m}$ (3072 triangles). See Figure 3 (b). In Figure 3(a), we can observe the Donald dual mesh with the control volumes for the coarsest case.
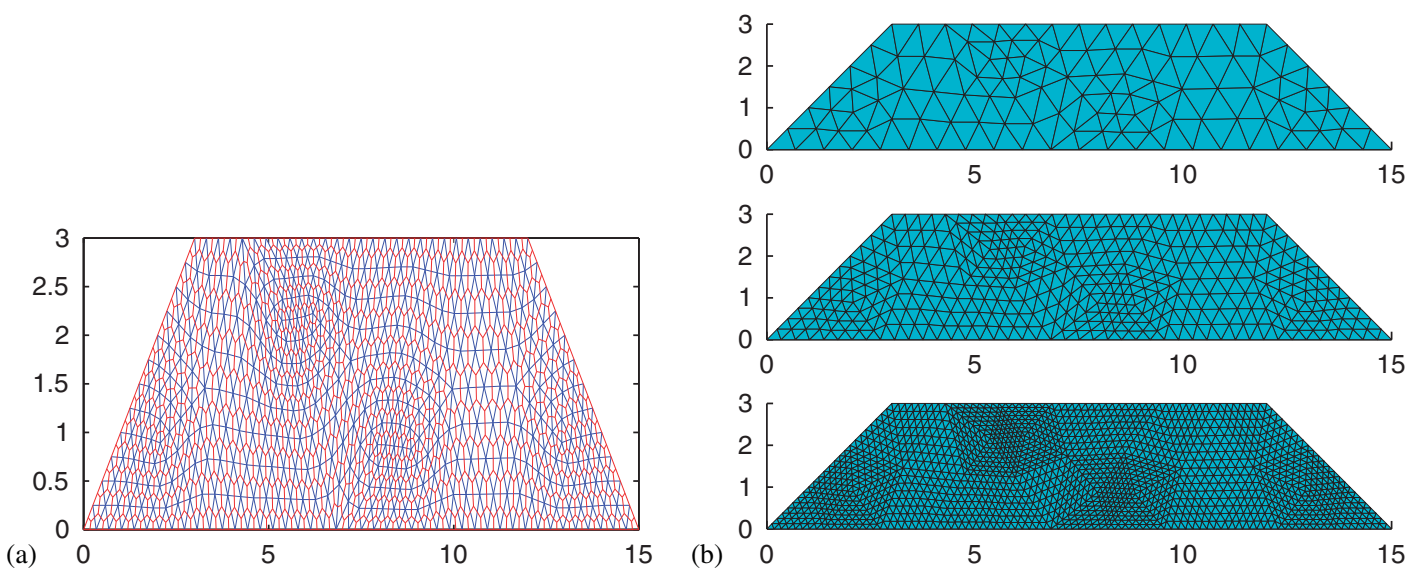

Figure 3. Spacial discretization mesh: (a) Donald dual mesh and (b) grid refinement. 

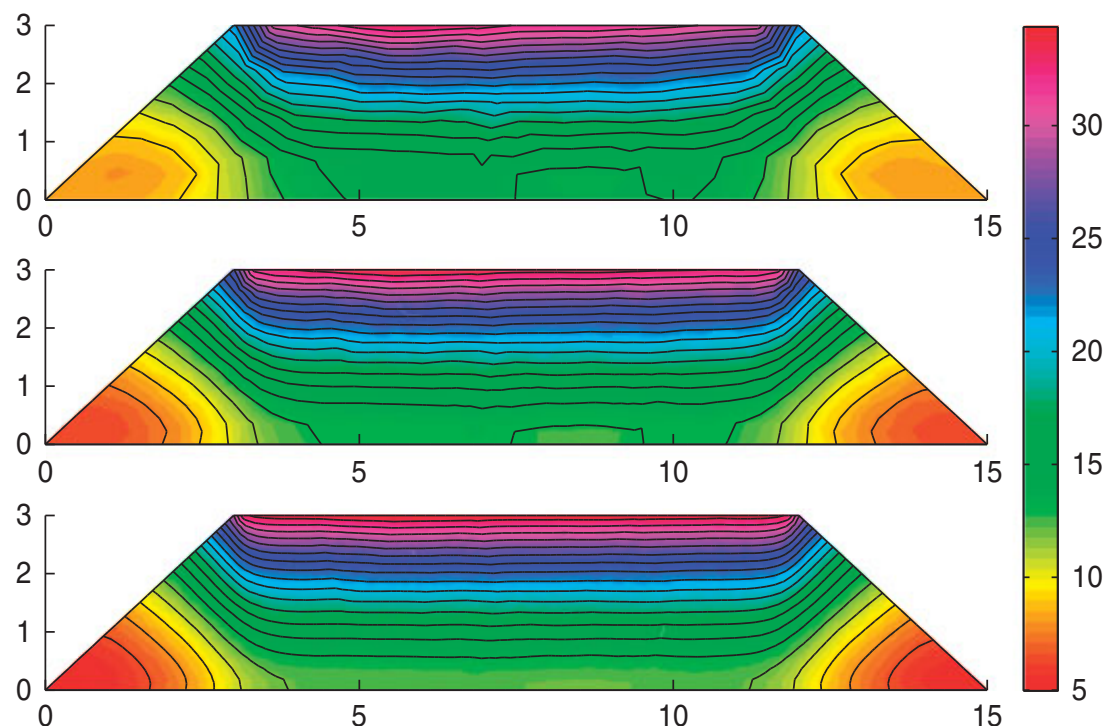

30

Figure 4. The acid concentration in the heap on the ninth day $(t=9)$ for three different refinements of the mesh.

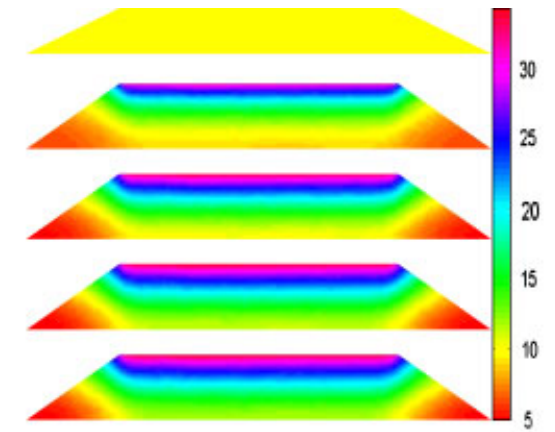

(a)

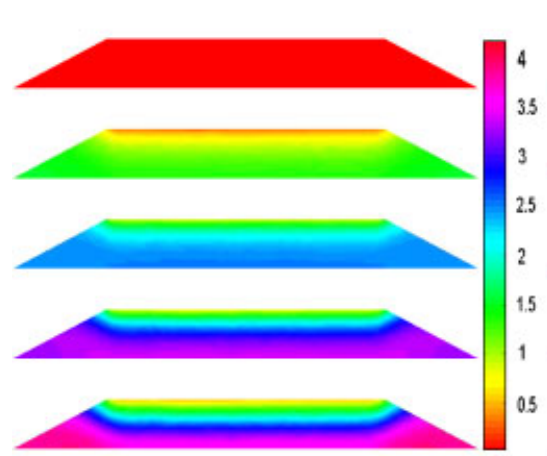

(b)

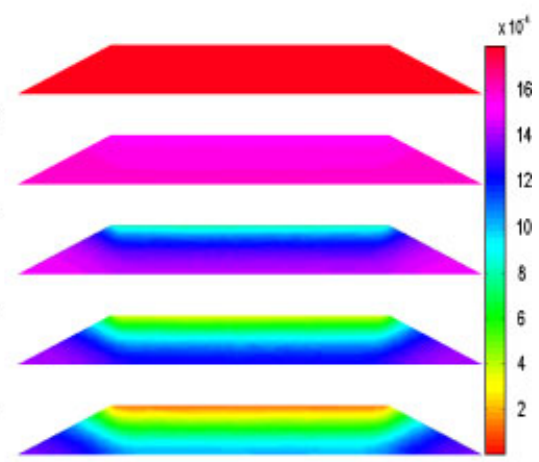

(c)

Figure 5. The first 5 days of evolution for the finest grid: (a) acid concentration; (b) liquid copper concentration; and (c) solid copper concentration.

Figure 4 shows the acid concentration evolution in the heap on the ninth day $(t=9)$, for the three first refinement of the mesh. We show 30 level curves, and we observe the smoothing of the curves with increasing refinement of the mesh, in agreement with the proved convergence of the method.

In Figure 5, we show the evolution during the first 5 days of the heap leaching process, for the acid (Figure 5(a)), the liquid copper (Figure 5(b)) and the solid copper (Figure 5(c)), considering the finest mesh.

Numerically, we observe that the acid penetrates fast into the heap, being still concentrated at the top of the heap leaching, despite the lapse of the day (Figure 5(a)). Note also that the acid concentration is lower at the extremes of the heap bottom. On the other hand, we see in Figure 5(a) that the copper in solution is enriching through the oxidation process (b), which increases the concentration solution copper in the ore (c). Finally, we note that in the fifth day the concentration of both solution and the ore is higher in copper at the bottom of the heap, exactly where the acid is rarefied. 


\section{Conclusions}

A two-dimensional mathematical model was developed and implemented to describe the leaching of copper tailings using sulfuric acid as a leaching agent. The model allows the leaching phenomenon to be described for different types of flow patterns for the leaching solution, and, particularly, a pattern established by the feeding system of the leaching solution, and the recovery of the copper-bearing solution at the bottom.

Each of the physico-chemical phenomena occurring during the leaching process was identified and analyzed. These phenomena may be summarized as follows: transport of sulfuric acid, reaction between the acid and the copper ore particles present in the solid matrix and transport of the copper solution. The acid is then usually further processed by solvent extraction and electrowinning. Mathematically, the model leads to two decoupled problems: the so-called model problem A, consisting of a degenerate convectiondiffusion equation for a quantity equivalent to the volumetric concentration of the solvent (sulfuric acid) and model problem $B$, which consists of a similar degenerate convection-diffusion equation for the copper concentration in the liquid, which is coupled with an ODE describing the copper transport in the solid phase.

Thus, we present here a convergence analysis for a vertex-centered finite volume scheme as a numerical approximation of the system of equations. The convergence proof in $L^{2}$-strong includes the degenerate case. Finally, we give some numerical results in accordance with the engineering literature examples $[1,3,7]$.

The results show that the model and its numerical approximation predict in a satisfactory manner the major trends exhibited by the phenomena studied, that is the evolution in time of the acid concentration and copper concentration in the liquid solution removed from the tailings. In addition, the model gives, for the entire domain, the spatial distribution of the acid concentration and copper concentration in the solution and the copper concentration in the solid phase.

\section{Acknowledgements}

M. S. has been supported by Fondecyt projects \# 1070694,\# 7070129, FONDAP and BASAL projects CMM, Universidad de Chile, and $\mathrm{Cl}^{2} \mathrm{MA}$, Universidad de Concepción. The work of I. S. P. was supported by the Dutch government through the national program BSIK: knowledge and research capacity, in the ICT project BRICKS (http://www.bsik-bricks.nl), theme MSV1, as well as the International Research Training Group NUPUS. E. C. has been supported by Universidad Católica de Temuco (Convenio de Desempeño DGI-CD2006-2-03), and F. C. was supported by the Mineral Technology Center Cettem Ltd.

\section{References}

1. Muñoz JF, Rengifo $P$, Vauclin M. Acid leaching of copper in a saturated porous material: parameter identification and experimental validation of a two-dimensional transport model. Journal of Contaminant Hydrology 1997; 27:1-24.

2. Casas JM, Martínez J, Moreno L, Vargas T. Bioleaching model of a copper-sulfide ore bed in heap and dump configurations. Metallurgical and Materials Transactions B 1998; 29:899-909.

3. Li J. Modelling of multiphase multicomponent flow and transport in heap leaching of copper ores. Master Thesis, WAREM, University of Stuttgart, Stuttgart, Germany, 2005.

4. Orr S. Enhanced heap leaching—part 1: insights. Mining Engineering 2002; 54:49-55.

5. Van Zyl DJA, Hutchison IPG, Kiel JE. Introduction to Evaluation, Design and Operation of Precious Metal Heap Leaching Projects. Society of Mining Engineering, Inc.: CO, 1988.

6. Domić E. Hidrometalurgia: Fundamentos, Procesos y Aplicaciones. Andros Impresores Ltda.: Santiago de Chile, 2001.

7. Suazo A. Lixiviación ácida en pilas de relave aglomerado. Tesis de Doctorado en Ciencias de la Ingeniería, Escuela de Ingeniería, Pontificia Universidad Católica de Chile, 2003.

8. Cariaga E, Concha F, Sepúlveda M. Simultaneous modeling of liquid and gaseous phases in heap leaching for copper production. In Proceedings of the III International Copper Hydrometallurgy Workshop, Menacho JM, Casas de Prada JM (eds), 23-25 November 2005. Santiago: Chile, 2005; 301-315.

9. Cariaga E, Concha F, Sepúlveda M. Convergence of a MFE-FV method for two phase flow with applications to heap leaching of copper ores. Computer Methods in Applied Mechanics and Engineering 2007; 196:2541-2554.

10. Radu FA, Pop IS, Attinger S. Analysis of an Euler implicit-mixed finite element scheme for reactive solute transport in porous media. Numerical Methods for Partial Differential Equations 2009; DOI: 10.1002/num.20436.

11. Radu FA, Pop IS. Newton method for reactive solute transport with equilibrium sorption in porous media. Journal of Computational and Applied Mathematics 2009; DOI: 10.1016/j.cam.2009.08.070.

12. Cariaga E, Concha F, Sepúlveda M. Flow through porous media with applications to heap leaching of copper ores. Chemical Engineering Journal 2005; 111:151-165.

13. Mellado ME, Cisternas LA. An analytical-numerical method for solving heap leaching of one or more solid reactants from porous pellets. Computers and Chemical Engineering 2008; 32:2395-2402.

14. Mellado ME, Cisternas LA, Gálvez ED. An analytical approach to heap leaching. Hydrometallurgy 2009; 95:33-38.

15. Kačur J, Van Keer R. Solution of contaminant transport with adsorption in porous media by method of characteristics. Mathematical Modelling and Numerical Analysis 2001; 35:981-1006.

16. Freundlich H. Colloid and Capillary Chemistry. Methuen: London, 1926.

17. Langmuir I. The adsorption of gases on plane surfaces of glass, mica and platinum. Journal of the American Chemical Society 1918; 40:1361-1403.

18. Afif M, Amaziane B. Convergence of finite volume schemes for a degenerate convection-diffusion equation arising in flow in porous media. Computer methods in Applied Mechanics and Engineering 2002; 191:5265-5286.

19. Ohlberger M. A posteriori error estimates for vertex centered finite volume approximations of convection-diffusion-reactions equations. Mathematical Modelling and Numerical Analysis 2001; 35:355-387. 
20. Bank RE, Rose DJ. Some error estimates for the box method. SIAM Journal on Numerical Analysis 1987; 24:777-787.

21. Hackbusch W. On first and second order box schemes. Computing 1989; 41:277-296.

22. Cai ZQ, Mandel J, McCormick S. The finite volume element method for diffusion equations on general triangulations. SIAM Journal on Numerical Analysis 1991; 28:392-402.

23. Forsyth PA. A control volume finite element approach to NAPL groundwater contamination. SIAM Journal on Scientific and Statistical Computing 1991; 12:1029-1057.

24. Radu FA, Sepúlveda M, Pop IS, Vera Villagran OP. Error estimates for the finite volume discretization for the porous medium equation. Journal of Computational and Applied Mathematics 2009; DOI: 10.1016/j.cam.2009.08.071.

25. Bear J. Dynamics of Fluids in Porous Media. Dover: New York, 1988.

26. Helmig R. Multiphase Flow and Transport Processes in the Subsurface: A Contribution to the Modeling of Hydrosystems. Springer: Berlin, Heidelberg, 1997.

27. Bastian P. Numerical computation of multiphase flow in porous media. Habilitationsschrift, Christian-Albrechts-Universitat Kiel, 1999.

28. Cariaga E, Concha F, Sepúlveda M. Flow and transport in leaching heaps: application of the theory of multiphase flow through porous media. In Proceedings of the IV International Copper Hydrometallurgy Workshop, Menacho JM, Casas de Prada JM (eds), 16-18 May 2007. Santiago: Chile, 2007; 255-261.

29. Cariaga E. Mathematical modelling of copper heap leaching. Thesis of Doctor in Applied Sciences, Department of Mathematical Engineering, University of Concepción, 2008. Available from: http://www.ing-mat.udec.cl/.

30. James F, Sepúlveda M, Valentin P. Statistical thermodynamics models for a multicomponent two-phases equilibrium isotherm. Mathematical Models and Methods in Applied Sciences 1997; 7:1-29.

31. Remešíková M. Numerical solution of direct and inverse contaminant transport problems with adsorption. Ph.D. Thesis, Univerzita Komenského Bratislava, 2005.

32. Alt HW, Luckhaus S. Quasilinear elliptic-parabolic differential equations. Mathematische Zeitschrift 1983; 183:311-341.

33. Chavent G, Jaffré J. Mathematical Models and Finite Elements for Reservoir Simulations. North-Holland: Amsterdam, 1978.

34. Carrillo J. Entropy solutions for nonlinear degenerate problems. Archive for Rational Mechanics and Analysis 1999; 147:269-361.

35. Hornung $U$, Jäger W, Mikelić A. Reactive transport through an array of cells with semipermeable membranes. RAIRO Modélisation Mathématique et Analyse Numérique 1994; 28:59-94.

36. Knabner $\mathrm{P}$, Otto $\mathrm{F}$. Solute transport in porous media with equilibrium and nonequilibrium multiple-site adsorption. Nonlinear Analysis, TMA 2000; 42:381-403.

37. Van Duijn CJ, Knabner P. Solute transport in porous media with equilibrium and nonequilibrium multiple-site adsorption: travelling waves. Journal für die Reine und Angewandte Mathematik 1991; 415:1-49.

38. Bouillard N, Eymard R, Herbin R, Montarnal P. Diffusion with dissolution and precipitation in a porous medium: mathematical analysis and numerical approximation of a simplified model. ESAIM: Mathematical Modelling and Numerical Analysis 2007; 41:975-1000.

39. Van Duijn CJ, Pop IS. Crystal dissolution and precipitation in porous media: pore scale analysis. Journal für die Reine und Angewandte Mathematik 2004; 577:171-211.

40. Devigne VM, Pop IS, van Duijn CJ, Clopeau T. A numerical scheme for the pore scale simulation of crystal dissolution and precipitation in porous media. SIAM Journal on Numerical Analysis 2008; 46:895-919.

41. Eymard R, Gallouët T, Herbin R. In Finite Volume Methods, Ciarlet PG, Lions JL (eds). Handbook of Numerical Analysis, vol. VII. Elsevier Science B.V: Amsterdam, 2000.

42. Kröner D. Numerical Schemes for Conservation Laws. Wiley-Teubner: Stuttgart, 1997.

43. Ciarlet PG. The Finite Element Method for Elliptic Problems. North-Holland: Amsterdam, 1978.

44. Eymard R, Gallouët T, Herbin R, Michel A. Convergence of a finite volume scheme for nonlinear degenerate parabolic equations. Numerische Mathematik 2002; 92:41-82.

45. Teman R. Navier-Stokes Equations, Theory and Numerical Analysis (3rd edn). North-Holland: Amsterdam, 2001. Reprinted in the AMS Chelsea series, AMS, Providence.

46. Yosida K. Functional Analysis. Springer: Berlin, 1965. 